\title{
MicroRNA-141 enhances anoikis resistance in metastatic progression of ovarian cancer through targeting KLF12/Sp1/survivin axis
}

Celia S. L. Mak', Mingo M. H. Yung ${ }^{1}$, Lynn M. N. Hui ', Leanne L. Leung ${ }^{1}$, Rui Liang ${ }^{1}$, Kangmei Chen', Stephanie S. Liu', Yiming Qin², Thomas H. Y. Leung ${ }^{1}$, Kai-Fai Lee', Karen K. L. Chan', Hextan Y. S. Ngan' and David W. Chan ${ }^{1 *}$

\begin{abstract}
Background: Cancer metastasis is determined by the formation of the metastatic niche and the ability of cancer cells to adapt to microenvironmental stresses. Anoikis resistance is a fundamental feature of metastatic cancer cell survival during metastatic cancer progression. However, the mechanisms underlying anoikis resistance in ovarian cancer are still unclear.

Methods: Expressions of miRNA-141 and its downstream targets were evaluated by qPCR, Western blotting, Immunohistochemical (IHC) and in situ hybridization (ISH) assays. The luciferase assays were used to prove KLF12 as the downstream target of miR-141. The CDNA microarray and apoptotic protein arrays were used to identify the targets of miR-141 and KLF12. The competition of KLF12 and Sp1 on survivin promoter was examined by ChIP assay. IHC analysis on ovarian cancer tissue array was used to evaluate the expressions of KLF12 and miR-141 and to show the clinical relevance. The functional studies were performed by in vitro and in vivo tumorigenic assays.

Results: Enforced expression of miR-141 promotes, while knockdown of miR-141 expression inhibits, cell proliferation, anchorage-independent capacity, anoikis resistance, tumor growth and peritoneal metastases of ovarian cancer cells. Bioinformatics and functional analysis identified that Kruppel-related zinc finger protein AP-2rep (KLF12) is directly targeted by miR-141. Consistent with this finding, knockdown of KLF12 phenocopied the effects of miR-141 overexpression in ovarian cancer cells. In contrast, restoration of KLF12 in miR-141-expressing cells significantly attenuated anoikis resistance in ovarian cancer cells via interfering with Sp1-mediated survivin transcription, which inhibits the intrinsic apoptotic pathway and is crucial for ovarian cancer cell survival, anoikis resistance and peritoneal metastases. Immunohistochemical (IHC) and in situ hybridization (ISH) assays confirmed that miRNA-141 expression is inversely correlated with KLF12 expression and significantly associated with advanced ovarian cancers accompanied with distal metastases, underscoring the clinical relevance of our findings.
\end{abstract}

Conclusions: Our data identify a novel signaling axis of miR-141/KLF12/Sp1/survivin in enhancing anoikis resistance and likely serves as a potential therapeutic target for metastatic ovarian cancer.

Keywords: miR-141, Anoikis resistance, Ovarian cancer, KLF12, Sp1, survivin

\footnotetext{
* Correspondence: dwchan@hku.hk

'Department of Obstetrics and Gynaecology, L747 Laboratory Block, LKS

Faculty of Medicine, The University of Hong Kong, 21 Sassoon Road, Hong

Kong SAR, People's Republic of China

Full list of author information is available at the end of the article
} 


\section{Background}

Epithelial ovarian cancer (EOC) is the most lethal gynecological malignancy worldwide. This disease is generally called the "silent killer" because there are no symptoms, and thus, the majority of patients are diagnosed when the cancer is at an advanced stage with extensive micrometastases [1]. Most deaths from this cancer are attributed to metastatic progression [1]. Therefore, understanding the molecular mechanisms of metastases may assist in the development of targeted therapies to improve the outcomes of this disease. Cancer metastasis is determined by the formation of the metastatic niche and the ability of cancer cells to adapt to the microenvironment [2]. However, the associated molecular mechanisms remain unclear.

Metastasis is a multistep process that allows cancer cells to spread from the primary site to a distant location in the body and includes local invasion, intravasation, circulation, extravasation, proliferation and angiogenesis [3]. Among the steps that occur during cancer cell metastasis, escaping apoptosis (i.e., anoikis resistance) while detaching from the extracellular matrix or neighboring cells at the primary sites and circulating in transit to a distant location is of utmost importance. Many studies have suggested that the upregulation of oncogenes or the loss of tumor suppressors in the signaling pathways associated with the death receptor (extrinsic), mitochondrial (intrinsic) and convergence pathways (IAPs) allows tumor cells to circumvent anoikis to evade restriction by integrin-ECM interactions [4]. Unlike other cancers, peritoneal metastases is the most common route of ovarian cancer metastasis [5]. But similar to other cancers, micrometastases is the commonly cause of ovarian cancer recurrence as well as chemoresistance [6]. The detached ovarian cancer cells acquire anoikis resistance to survive in the ascitic fluid prior to metastatic colonization in the omentum/peritoneum is a vital trait of metastatic progression $[7,8]$.

Emerging evidence has indicated that deregulation of miRNAs is involved in cancer metastases [9]. MicroRNAs (miRNA) are small endogenous, non-coding RNAs of 21 to 25 nucleotides $[10,11]$ that negatively regulate gene expression by translational repression or endonucleolytic cleavage of the target mRNAs $[12,13]$ at the post-transcriptional level. Since 2002, emerging evidence has suggested that the aberrant expression of miRNAs in human cancers may modulate tumor progression by regulating tumor suppressors and oncogenes [14]. For example, miR-221/222 has been shown to increase chemoresistance in breast cancer [15], increase tumorigenicity of cancer cells through PTEN [16], promote human castration-resistant prostate cancer development [17] and stimulate invasion in glioblastoma [18]. Another example is the miR-200 family, which facilitates endometrioid carcinoma growth by modulating the Notch, p53, MAPK, and ErbB signaling pathways $[19,20]$. However, the functional roles of miRNAs associated with anoikis resistance in human ovarian cancer have rarely been investigated.

In this study, we found that a member of the miR-200 family, miR-141, is prominently overexpressed in advanced and metastatic ovarian cancers. Using a series of functional and biochemical analyses, we identified that miR-141 enhances resistance against microenvironmental stresses and anoikis by targeting and repressing KLF12 expression, which allows Sp1 to upregulate survivin expression, an inhibitor of intrinsic apoptotic pathway, is crucial in promoting cell survival, proliferation and peritoneal metastases of ovarian cancer.

\section{Methods}

\section{Cell culture and human tissue samples}

The human ovarian cancer cell lines; SKOV3 (purchased from American Type Culture Collection, ATCC), OVCA433 and A2780cp and cervical cancer cell lines (OV2008 and C13*) (kindly provided by Prof. B. Tsang, Department of Obstetrics and Gynecology, University of Ottawa) were used in this study. They were grown either in Dulbecco's modified Eagle's medium (DMEM) or in minimum essential medium (MEM) (Invitrogen, Grand Island, N.Y., USA) supplemented with $10 \%$ fetal bovine serum (FBS) (Invitrogen) and 1\% penicillin-streptomycin $(\mathrm{P} / \mathrm{S})$ (Invitrogen). Three human immortalized ovarian surface epithelial cell lines, HOSE 17-1, HOSE 11-12 and HOSE 96-9-10 (kindly provided by Prof. GSW. Tsao, The University of Hong Kong) [21], were cultured in Medium 199 and Medium 105 (Sigma-Aldrich Corp., St. Louis, MO, USA) supplemented with 10\% FBS and 1\% $\mathrm{P} / \mathrm{S}$. All cells were incubated in an incubator containing $5 \% \mathrm{CO}_{2}$ at $37{ }^{\circ} \mathrm{C}$. In-house STR DNA profiling analysis was used to authenticate the cell lines, and mycoplasma contamination was assessed. All clinical samples (49 ovarian cancer tissues and 12 normal ovarian tissues) were obtained from the Department of Obstetrics and Gynecology of The University of Hong Kong at Queen Mary Hospital. The specimens were snap-frozen immediately after collection and were stored at $-80{ }^{\circ} \mathrm{C}$ until use.

\section{Plasmids and cell transfection}

The miR-141-expressing construct (pmR-ZsGreen1miR-141) (Forward -TTGAGCTGAAAGCTTTGCACA AGG, Reverse-AAGTGACTTGGATCCG, 583 bp) was generated by PCR amplification and ligated into the pmR-ZsGreen1 plasmid (Clontech, Mountain View, CA). The KLF12-expressing construct (pCMV6-KLF12) and Sp1-expressing construct (pCMV6-Sp1) were purchased from Origene (Origene Technologies, Inc., Rockville, M.D., USA). The Ambion ${ }^{\odot}$ Anti-miR ${ }^{\mathrm{Tm}}$ miRNA 
Inhibitor targeting miR-141 was purchased from ThermoFisher (ThermoFisher Scientific, Waltham, MA, USA). A TriFECTa ${ }^{\circ}$ Kit with 3 siRNAs (KD1: NM_007249 duplex 1, KD2: NM_007249 duplex 2, KD3: NM_007249 duplex 3) and shRNA (NM_007249.4) targeting KLF12 were purchased from Integrated DNA Technologies (IDT, Commercial Park, Coralville, IA, USA) and GeneCopoeia (GeneCopoeia, Inc., USA). The universal negative control RNA duplex (NC1), scrambled siRNA control and shRNA scrambled control clone for psi-HIV-U6 were used as the negative controls. For luciferase reporter assays, pairs of oligonucleotides containing the 3'UTR binding site for miR-141 were purchased from IDT. Cell transfection was performed using Lipofectamine $^{\mathrm{TM}} 2000$ (Invitrogen) according to the manufacturer's instructions. Stable clones overexpressing miR-141 were harvested after approximately 2 weeks of G418 selection. The transiently silenced KLF12 cells were collected at $48 \mathrm{~h}$ post-transfection, and lentivirus was used to infect cancer cells with KLF12 shRNA.

\section{RNA extraction and quantitative real-time PCR}

TRIzol reagent (Invitrogen, Life Technologies) was used to extract total RNA from cell lines and primary cancer tissue samples. Twenty-five nanograms of total RNA was used as a template to generate first-strand cDNA with miRCURY LNA ${ }^{\mathrm{m}}$ Universal RT microRNA PCR using a Universal cDNA Synthesis Kit (Exiqon, Vedbaek, Denmark). Quantitative real-time PCR (QPCR) was performed using a miRCURY LNA $^{\mathrm{TM}}$ Universal RT miRNA PCR Kit and SYBR Green master mix (Exiqon, Denmark) in an ABI 7500 system (Applied Biosystems). The miR141 probes (product no. 204504) and SNORD48, the reference gene (product no. 203903), were purchased from Exiqon. Each sample was assayed in triplicate, and the relative expression of miR-141 was normalized to SNORD48.

\section{Cell viability assays, cell migration and anoikis assays} Cell viability was measured by XTT assays, foci formation assays and soft agar assays. For the XTT assay, approximately 1000-2000 cells were seeded into each well of a 96-well plate. The full serum medium was changed to $1 \%$ serum medium after cell seeding for $24 \mathrm{~h}$. Cell viability was measured using a Cell Proliferation Kit II (Roche Biosciences, Indianapolis, IN, USA) according to the manufacturer's instructions. To perform the foci formation assay, cells were seeded in 6-well plates at a density of 800 cells/well. After seeding the cells for $24 \mathrm{~h}$, the medium was exchanged with $1 \%$ serum medium. After approximately 1-2 weeks, the colonies that formed were fixed with $70 \%$ ethanol for $30 \mathrm{~min}$ before staining with $20 \%$ Giemsa stain (Sigma). The foci were counted. The soft agar assay was used to examine the anchorage- independent growth of ovarian cancer cells. Cancer cells with a density ranging from 500 to 1000 cells/well were embedded in a $0.6 \%$ DMEM-agarose layer on top of a $2 \%$ DMEM-agarose base. After 14 to 21 days, the viable colonies with $>20$ cells were counted. The cell migratory capacity of ovarian cancer cells was examined by the Transwell cell migration assay kits (Chemicon International, Inc., Temecula, CA) according to the manufacturer's instructions. The migratory cells on transwell filters were visualized counted by bright-field microscopy (TE300, Nikon). For the anoikis assay, $1 \times 10^{6}$ cells were cultured in the wells of 6-well low-attachment plates pre-coated with $1 \%$ agarose gel. After $48 \mathrm{~h}$ of suspension, cells were harvested for cell death analysis by TUNEL assays and flow cytometry. Cleavage of PARP and cleavage of caspase 3 were evaluated by western blotting. The results were generated from more than three separate experiments and are presented as the mean $\pm \mathrm{SD}$.

\section{Computational miRNA target prediction and RNA gene expression profiling}

Three online programs, TargetScan 6.0 (http://www.targetscan.org/), PicTar (http://pictar.mdc-berlin.de/) and DIANA LAB (DIANA-microT web server v5.0, http:// diana.imis.athena-innovation.gr/DianaTools/index.php? $\mathrm{r}=$ MicroT_CDS/index), were used to identify potential targets of miR-141. For the gene expression profiling, total RNA was extracted from KLF12 knockdown OVCA433 cells and the vector control cells. $\mathrm{OD}_{260 / 280}$ ratios (1.7-2.1) and RNA integrity number $(\mathrm{RIN}>0.8)$ of the RNA samples were analyzed by a spectrophotometer and bioanalyzer, respectively. After verifying the quality, the samples were subjected to Affymetrix GeneChip processing (Human Genome U133 Plus 2.0 Array) in the Centre for Genomic Science, The University of Hong Kong. The GeneChip experiment labels RNA targets and hybridizes targets to the arrays, followed by stringent washes, staining and scanning. The data were analyzed by GeneSpring 12.6 (Agilent Technologies, Santa Clara, USA).

\section{Sp1 binding site prediction, Chromatin immunoprecipitation (ChIP) and Luciferase reporter assays}

Sp1 motif (MA0079.3) in JASPAR CORE Vertebrata database is represented in terms of the Position Weight Matrix (PWM), as annotated in the TRANSFAC ${ }^{\oplus}$ database. It was used to scan Sp1 binding sites (BS) in BIRC5 promoter, +/-1 $\mathrm{k}$ to TSS, chr17:76209278-76211277 (based on hg19 genome assembly), and ten putative BS were found. Five of them are covered by Sp1 ChIP-Seq peaks in 2 studies from ENCODE (Sp1 ChIP-Seq in A549 and HCT116), and two of the five are even covered by Sp1 ChIP-Seq peaks in 3 more studies from ENCODE (Sp1 ChIP-Seq in GM12878, H1-hESC and HepG2). 
The Chromatin immunoprecipitation (ChIP) Assay Kit (Millipore, Billerica, MA, USA) was used to investigate the competition binding of Sp1 and KLF12 on human survivin promoter. The immunoprecipitated chromatin was examined by PCR and the ChIP primers targeted the survivin promoter is shown (Additional file 1: Figure S3).

Luciferase constructs were generated by ligating oligonucleotides containing wild-type (KLF12, sense: 5'-CTC CCCTTCACAGTGTTAACACAAAAGGGC ATCACCA TCCCACGATGTCTGT-3'; antisense: 5'-CTAGACAGAC ATCGTG GGATGGTGATGCCCTTTTGTGTTAACAC TGTGAAGGGGAGAGCT-"; E2F3, sense: 5'-CATGGA ACCAGAACATCTGTCATGCAGTGTTGTCCCTT CCT ACCTTCTTCCT-3'; antisense: 5'-CTAGAGGAAGAAGG TAGGAAGGG ACAACACTGCATGACAGATGTTCTG GTTCCATGAGCT-3'; MAP2K4 sense: 5'-CGT AATA CCTGATTGATCACACAGTGTTAGTGCTGGTCAGAG AGACCTCATT-3'; antisense: 5'-CTAGAATGAGGTCT CTCTGACCAGCACT AACACTGTGTGATCAATCAG GTATTACGAGCT-3') or mutant (KLF12, sense: 5'-CT CCCCTTCACAGAGAAAACACAAAAGGGCATCACC ATCCCA CGATGTCTGT-3'; antisense: 5'-CTAGACA GACATCGTGGGATGGTGATGC CCTTTTGTGTTTT CTCTGTGAAGGGGAGAGCT-3') 3'UTR binding sites of miR-141 into the multi-cloning region of the pmirGLO Dual-Luciferase miRNA Target Expression Vector (Promega, Madison, WI, USA). HEK-293 cells were seeded into a 24-well plate and co-transfected with $100 \mathrm{ng}$ pmirGLO wild-type or mutant plasmids together with the pmR-141 plasmid and the pmR-ZsGreen 1 empty vector as a control (400 ng in total) using Lipofectamine ${ }^{\mathrm{TM}} 2000$ (Invitrogen). Luciferase activity was detected with a Dualluciferase Assay Kit (Promega) 48 h post-transfection using a F-4500 fluorescence spectrophotometer (Promega). For the dose-dependent model, 200, 400 and $600 \mathrm{ng}$ of pmR-141 and constant amounts of the KLF12 plasmid (200 ng) were co-transfected into HEK-293 cells. Renilla luciferase activity was used as the reference to normalize transfection efficiency. All experiments were repeated three times.

\section{Western blotting and human apoptosis array}

Cells were harvested and lysed using lysis buffer (Cell Signaling Technology) containing protease inhibitors (Sigma) and phenylmethylsulfonyl fluoride (PMSF) (Sigma Chemical Co., St Louis, MO, USA). Equal amounts of protein were separated by $10 \%$ SDS-PAGE and then transferred to Immobilon-P Transfer Membranes (Millipore Corporation, Bedford, MA, USA). The membranes were pre-blotted in 5\% skim milk prior to incubation in $1 \%$ skim milk containing primary anti-Sp1 (1:500; Millipore Darmstadt, Germany), anti-KLF12 and anti-XIAP (1:1000; Santa Cruz Biotechnology, Inc., Santa Cruz, CA, USA), anti-cleaved-PARP, anti-cleaved-caspase3
(1:1000; Cell Signaling Technology, Inc., Danvers), antiDDK (1:1000) (OriGene Technologies, Rockville, MD) and anti- $\beta$-actin (1:10000; Sigma-Aldrich, St. Louis, MO) antibodies overnight. The membranes were incubated with horseradish peroxidase-conjugated goat anti-rabbit or anti-mouse IgG (Amersham) and visualized using $\mathrm{ECL}^{\mathrm{TM}}$ Western Blotting Detection Reagent (Amersham). Images were captured by Fuji Medical X-Ray Film (Fuji) and developed by the Fuji system. The Human Apoptosis Array Kit (R\&D Systems, Inc., USA) was used based on the manufacturer's instructions.

\section{Immunohistochemistry (IHC) and miRNA locked nucleic acid (LNA) in situ hybridization (ISH)}

In situ hybridization (ISH) was performed to validate miR141 expression in a commercial ovarian cancer tissue array (OVC1021) (5 normal/benign samples and 97 cases of ovarian cancer) (Pantomics Inc., CA, USA) using the miRCURY LNA ${ }^{\mathrm{TM}}$ microRNA ISH Optimization Kit 5 (FFPE) (Exiqon, Vedbaek, Denmark) as described in our previous study [22]. First, the tissue assay was deparaffinized and incubated for $40 \mathrm{~min}$ at $37{ }^{\circ} \mathrm{C}$ with $20 \mu \mathrm{g} / \mathrm{ml}$ proteinase $\mathrm{K}$. Then, the array was dehydrated followed by hybridization with a miR-141 probe (5'-DIG/CCATCTTTACCAGA CAGTGTTA/DIG-3', 1:500) overnight at $50{ }^{\circ} \mathrm{C}$. Next, antiDIG reagent (sheep anti-DIG-AP, 1:400) was added, and the slide was incubated for $60 \mathrm{~min}$ at room temperature. Then, AP substrate was freshly prepared and applied to the slide for a $2 \mathrm{~h}$ incubation at $30{ }^{\circ} \mathrm{C}$ in a humidifying chamber, avoiding the dark. Finally, a nuclear counterstain was applied, and the slides were mounted with mounting medium (Eukitt).

For the immunohistochemistry analysis, xylene and alcohol at different percentages were utilized for slide deparaffinization and rehydration. Slides were then immersed in sodium citrate buffer (pH6) and boiled for $20 \mathrm{~min}$. Inhibition of the endogenous peroxidase was carried out by applying $0.3 \%$ hydrogen peroxidase $\left(\mathrm{H}_{2} \mathrm{O}_{2}\right)$. The slides were incubated with an anti-KLF12 polyclonal antibody $(1: 600)$ at $4{ }^{\circ} \mathrm{C}$ overnight after blocking with $10 \%$ normal rabbit serum for $45 \mathrm{~min}$. A standard streptavidin-biotin-peroxidase complex method was used for staining, followed by counterstaining with Mayer's hematoxylin. The stained slides were reviewed by two independent investigators. The results were analyzed by light microscopy, and scores were given based on the intensity and the percentage of the stained tissues.

\section{In vivo studies}

For the intraperitoneal model, stable SKOV3 miR-141expressing clones, or A2780cp shSu knockdown clone and the scrambled controls $\left(2 \times 10^{6}\right)$ were intraperitoneally injected into 5 -week-old female BALB/cAnN nude 
mice (or $N=5$ per group for SKOV3 miR-141 or A2780cp shSu, respectively). All mice were sacrificed after 2 to 4 weeks, and intraperitoneal tumor nodules were extracted and weighed to compare relative tumor burden.

\section{Statistical analysis}

Statistical analysis was performed using SPSS 14.0 (SPSS). A receiver operating characteristic (ROC) curve was applied to determine the cut-off points for the ISH and IHC data. Student's $t$-test (for parametric data) and the Mann-Whitney test (for non-parametric data) were used. The results are expressed as the mean \pm SD from at least three independent experiments. The $\chi^{2}$ test or Fisher's exact test was used to analyze the association of miR-141 and KLF12 expression and the clinicopathological parameters. The Pearson correlation was used to show the inverse relationship of miR-141 and KLF12. $P<0.05$ was regarded as statistically significant.

\section{Results}

miR-141 is frequently upregulated in ovarian cancer

The loss of anchorage dependence is the main consequence of tumor cell escape from detachment-induced cell death (anoikis). Using miRCURY ${ }^{\mathrm{T} M}$ LNA Array profiling
(1264 miRNAs) analysis of two types of ovarian cancer cells $(+/-$ in the ability to form colonies in soft agar assays), we found 18 upregulated miRNAs in the cell lines that could form colonies in soft agar (Fig. 1a). Among these 18 miRNAs, 12 potential candidates with higher than 2-fold changes in the median log ratios were selected for further functional screening. Soft agar assays were performed with ovarian cancer cells ectopically expressing the selected premiRNAs. The results showed that miR-141 significantly increased the anchorage-independent growth of ovarian cancer cells. However, other miR-200 family members, such as miR-200b/200c/429 and miR-200a, also showed high expression levels in ovarian cancer cells (Fig. 1a) but had no obvious anchorage-independent effects (data not shown), indicating that only miR-141 of the miR-200 family is involved with promoting anoikis resistance in ovarian cancer cells. The miRNA microarray findings were confirmed by comparing HOSE cell lines and ovarian cancer cell lines. Real-time quantitative RT-PCR (qPCR) was employed to assess a panel of ovarian cancer cell lines (SKOV3, ES2, OVTOKO, A2780S, OV429, OVISE, OVCA433, RMG-1, A2780cp, OV420) and three immortalized HOSE cell lines (HOSE17-1, HOSE 96-9-10 and HOSE 11-12). Our data showed that miR-141 was frequently upregulated in the ovarian cancer cell lines

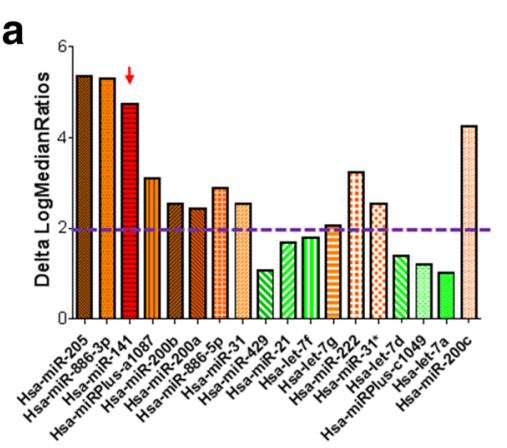

b

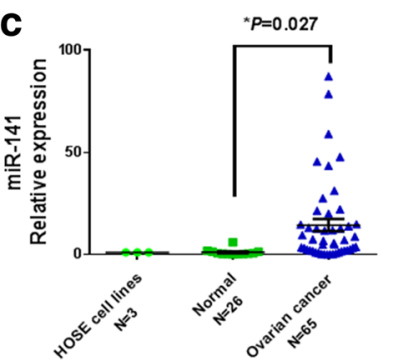

d
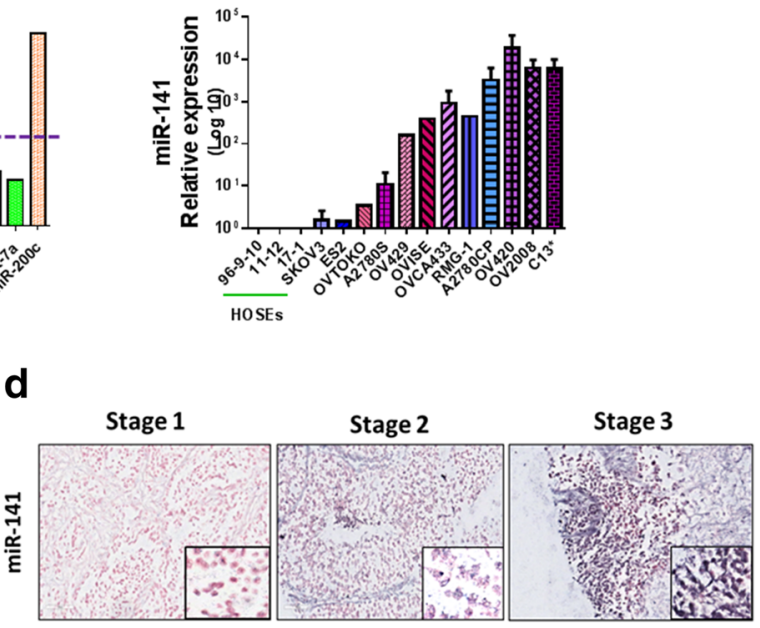

Fig. 1 Mir-141 is frequently upregulated in advanced ovarian cancers. a A graph showing 18 miRNAs with the highest expression in microRNA profiling analysis which was performed using the miRCURY LNA ${ }^{\mathrm{TM}}$ miRNA array (Exiqon) on two types of ovarian cancer cell lines; anchorage-growth independent (A2780s, A2780cp, SKOV3, C13*), and anchorage-growth dependent (HOSEs). The dot line shows the miRNAs with more than 2 folds of Delta LogMedianRatios were selected for further functional analysis using transfection of their pre-miRNA expressing plasmids in ovarian cancer cell lines e.g. A2780cp and SKOV3 and performing of soft agar assay. $\mathbf{b}$ Real time QPCR analysis showed that miR-141 is upregulated in ovarian cancer cell lines $(N=12)$ as compared with the immortalized cell lines, HOSEs, $(N=3)$. Samples were normalized with SNORD48. c Graphical representation of qPCR analysis showing that miR-141 is elevated in ovarian cancer $(N=65)$ when compare to the control normal ovary tissues $(N=26)$ and immortalized cell lines $(N=3)$. d Representative pictures showing miR-141 ISH expression signal (dark blue staining) is gradually increased along the tumor stages of epithelial ovarian cancer. (Magnification × 10; scale bar, $100 \mu \mathrm{m}$ ) 
$(N=12)$ and ovarian cancer clinical samples $(N=49)$ compared to either normal ovarian tissues $(N=12)$ or HOSE cells $(N=3)$ (Fig. 1b \& c). Furthermore, consistent with the findings in the cell lines, the mean expression level of miR-141 in clinical ovarian cancer samples $(N=49)$ was found to be $\sim 10$-fold higher than that of normal ovary tissues $(N=12)$ as well as the HOSE cell lines (Fig. 1c). In situ hybridization was then performed on a commercial human ovarian cancer tissue array (OVC1021, Pantomics), which further confirmed that the upregulation of miR-141 in advanced ovarian cancer is significantly correlated with cancer metastasis (Fig. 1d, Table 1). These findings suggest that upregulation of miR-141 is common in ovarian cancers, especially late-stage ovarian cancer.

\section{Increased miR-141 augments anchorage-independent growth and survival of ovarian cancer cells in vitro and tumor growth in vivo}

Upregulated miR-141 was strongly associated with advanced ovarian cancers, which is consistent with a previous study [23]. To confirm the oncogenic functions of miR-141, a miR-141 precursor-expressing plasmid (pmR-ZsGreen1 vector, Clontech) was stably transfected into three advanced ovarian cancer cell lines: A2780CP (p53 mutated) (141-C2 and 141-C8), OVCA433 (p53 wildtype) (141-C5 and 141-C10) and SKOV3 (p53 deleted) (141-C1 and 141-C4) (Fig. 2a, left panel). An ability to survive in the stressful microenvironment is one of the pre-requisites for the development of anoikis resistance in cancer cells. Therefore, we examined the cell viability of stable miR-141-expressing clones under low serum conditions (1\% FBS). Using XTT cell proliferation assays, we showed that all miR-141-expressing clones had significantly higher proliferation rates (3- to 4-fold) than the scrambled controls (Fig. 2b, left panel).

Table 1 Clinicopathological correlation of the expression of miR-141 in an ovarian cancer tissue array (OVC1021). The 5-fold cut-off was determined by ROC analysis

\begin{tabular}{lllll}
\hline Parameters & $\mathrm{n}(=97)$ & \multicolumn{3}{l}{ miR-141 expression } \\
\cline { 3 - 5 } & & $\leq 5$-fold & $>5$-fold & $p$ \\
\hline Grade & & & \\
$\quad$ Low $(1+2)$ & 50 & $24(48 \%)$ & $26(52 \%)$ & \\
$\quad$ High (3) & 46 & $24(52 \%)$ & $22(48 \%)$ & 0.838 \\
Stage & & & & \\
$\quad$ Early (1) & 48 & $31(65 \%)$ & $17(35 \%)$ & \\
Late (2+3) & 49 & $18(37 \%)$ & $31(63 \%)$ & $0.008^{*}$ \\
Metastasis & & & & \\
$\quad$ Yes & 24 & $5(21 \%)$ & $19(79 \%)$ & \\
No & 73 & $44(62 \%)$ & $29(38 \%)$ & $0.001^{*}$ \\
\hline *P $<0.05$ & & & &
\end{tabular}

Furthermore, foci formation assays also confirmed that stable miR-141-expressing clones of the three cell lines exhibited increased cell viability (Fig. 2c, left panel) under low serum (1\%) culture conditions. The ability to survive in unfavorable conditions provides advantages to cancer cells in acquiring anoikis resistance. To evaluate cancer cell resistance to anoikis, soft agar assays were used. Stable miR-141-expressing clones of A2780cp (141-C2 and 141-C8) and SKOV3 (141-C2 and 141-C3) were tested in this assay. Our results revealed that the clones A2780cp (141-C2 and 141-C8) exhibited a substantial increase in size ( 10-fold) and number (1.5- to 2-fold), and the SKOV3 stable clones (141-C2 and 141-C3) also exhibited an increase in size ( 2- to 4-fold) and number (1.5- to 2-fold) in soft agar compared with the vector controls (Additional file 2: Figure S1).

Then, miR-141 was inhibited by the anti-miR ${ }^{\mathrm{Tw}}$ miRNA Inhibitor (Ambion) in the miR-141-enriched OVCA433 and SKOV3 cells (Fig. 1b). The depletion of miR-141 in OVCA433 and SKOV3 (Fig. 2a, right panel) led to a significant reduction in cell viability by 38 to $45 \%$ (Fig. $2 \mathrm{~b}$, right panel) and the number of foci by 45 to $65 \%$ (Fig. 2c, right panel) in the ovarian cancer cells cultured in low serum medium. Taken together, these findings suggested that miR-141 plays a crucial role in mediating cell survival under stress condition in ovarian cancer cells.

\section{KLF12 is a direct target of miR-141 in ovarian cancer}

One miRNA can modulate a wide range of downstream targets; thus, we examined the potential downstream targets of miR-141 that may mediate these tumorigenic behaviors in ovarian cancer cells. Through an in silico study using three bioinformatics algorithms (PicTar, Miranda, and TargetScanHuman 6.0), we successfully sorted out 65 potential genes from the above 3 programs using gene ontology terms according to the Database for Annotation, Visualization and Integrated Discovery (DAVID) [24] (Fig. 3a). Ontology analysis using miRNA Targets database [25] identified that those potential targets are involved in metabolic process, biological regulation, localization and cellular process (Fig. 3b). Notably, some potential genes have been previously reported that they are the direct targets of miR-141 and are associated with cell proliferation and metastasis of human cancers. For examples, PAPP-A, a factor of metabolic process, is frequently found in invasive breast cancer [26, 27]; FUS, a binding protein, is associated with neuroblastoma's proliferation, migration and survival [28]; DLC1 is a wellknown tumor suppressor for a variety of human cancers [29]; as well as IRS2 is a suppressor of cell proliferation and invasion of thyroid cancer [30]. Interestingly, there is a potential target, SIK1, has previously reported to be associated with anoikis resistance and metastasis via interaction with LKB1 and in p53-dependent manner [31]. 


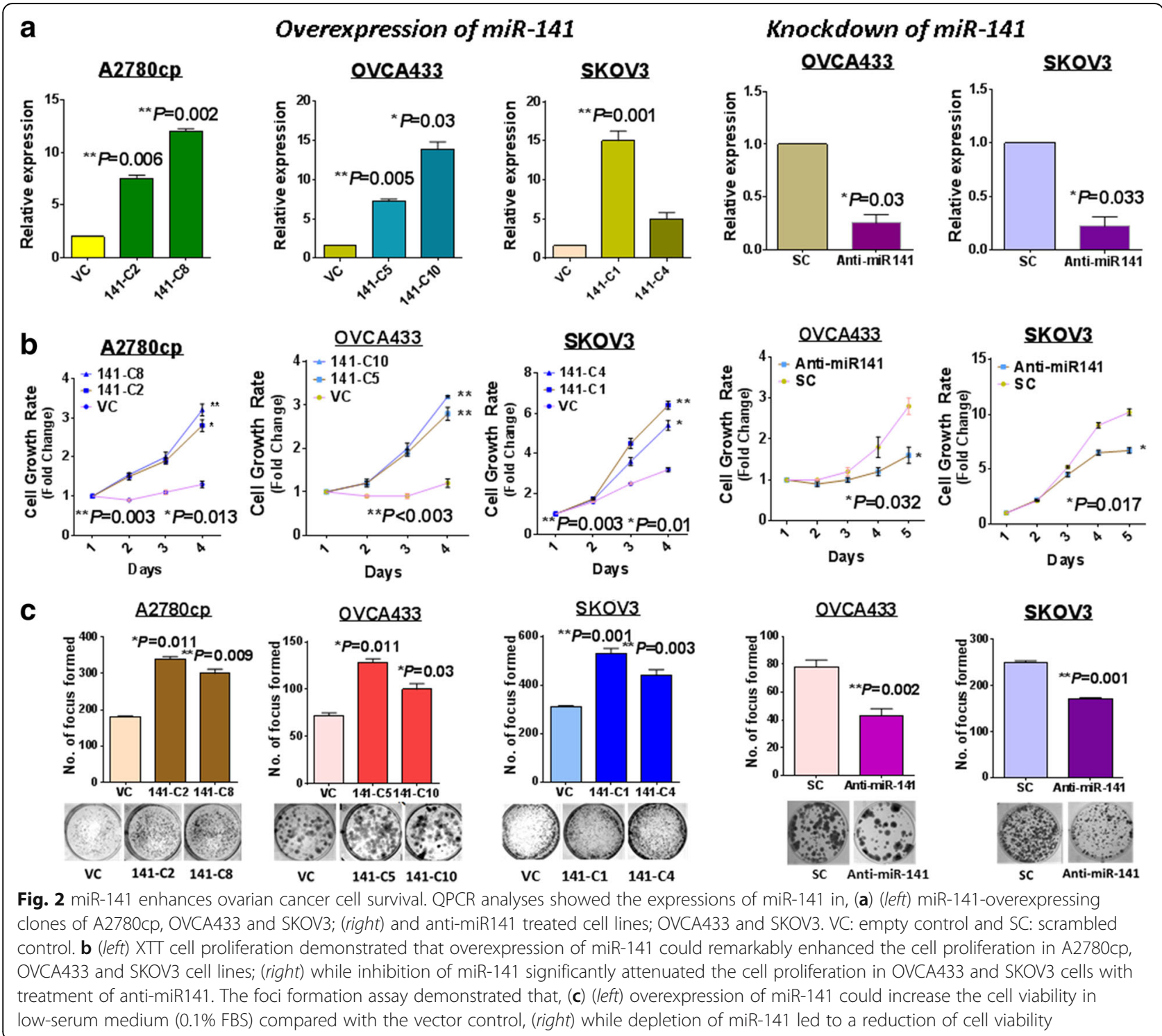

Likewise, our findings demonstrated that increased miR-141 significantly enhances the anoikis resistance of ovarian cancer cells. Among these cell lines, some cell lines, such as A2780cp and SKOV3, harboring mutated or deleted $\mathrm{p} 53$, suggesting that the increased anoikis resistance of ovarian cancer cells does not solely depend on SIK1/LKB1/p53 axis. To explore other putative targets associated with anoikis resistance in ovarian cancer cells, we next analyzed other potential targets which are involved in cell survival and have high score in algorithms prediction. In line with two known targets of miR-141; SIK1 and FUS, we also evaluated the expression of another three putative targets; E2F3, MAP2K4 and KLF12 (Fig. 3c). Our western blot analysis showed that overexpression of miR-141 and transfection of anti-miR141 could remarkably reduce and increase the expressions of SIK1 and KLF12, respectively (Fig. 3d). However, FUS, MAP2K4 and E2F3 just showed minor or even no change in the expressions (Fig. 3d). To further confirm these three putative targets was controlled by miR-141, the 3'UTR regions flanking the miR-141 binding sites of each target were subcloned into a luciferase reporter vector, pmirGLO, generating pmirGLOMAP2K4, pmirGLO-E2F3 and pmirGLO-KLF12. Luciferase reporter assays of cells co-transfected with miR-141 and the three target plasmids revealed that the E2F3 and MAP2K4 vectors only showed a $10 \%$ reduction in luciferase reporter activities $(P<0.005)$, while KLF12 showed a $35 \%$ reduction $(P<0.001)$ compared with the vector control (Additional file 3: Figure S2). This finding is in line with the above western blot analysis (Fig. 3d), indicating E2F3 and MAP2K4 may not the direct targets of miR-141. On the other hand, further analysis indicated that miR-141 could significantly reduce the pmirGLO-KLF12 luciferase activity in a dose-dependent manner $(P<0.035)($ Fig. 3e). 


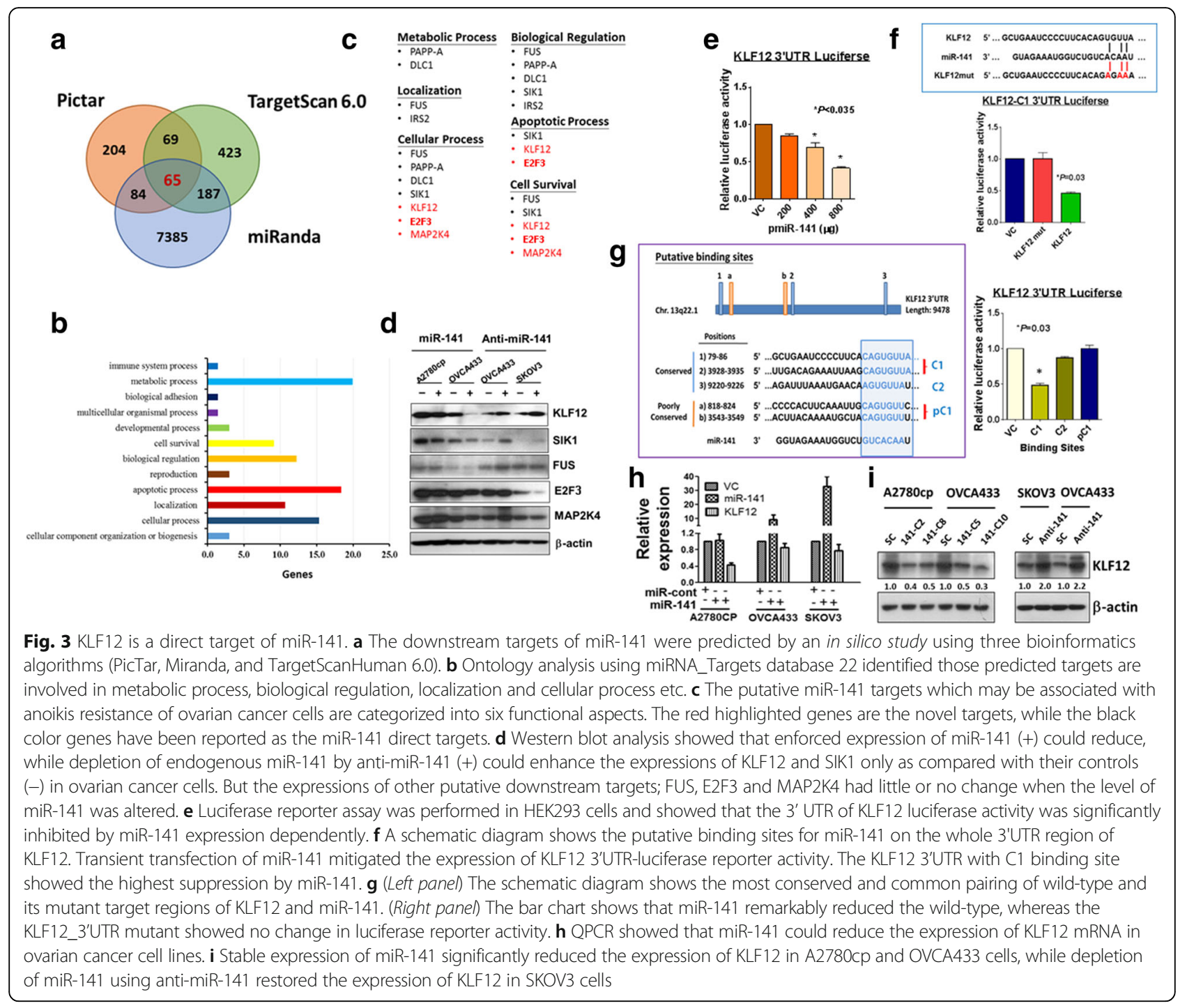

There are 3 conserved ( 2 types of miR-141 target sequence) and 2 poorly conserved (one type of miR-141 target sequence) miR-141 binding sites in the KLF12 3'UTR (Fig. 3f). Using luciferase reporter assays, we found that miR-141 could significantly attenuate the luciferase activity from the reporter fused to the $\mathrm{C} 1 \mathrm{miR}-141$ target sequence (GTCACAAT) of the KLF12 3'UTR by 51\% and the C2 miR-141 target sequence (TCACAAT) by $14 \%$, while there was no effect on the $\mathrm{pC} 1$ poorly conserved miR-141 target sequence (CAGTGTT) (Fig. 3g). Additionally, a point mutation (AACCAAT) in the miR-141 seed region in the 3'UTR (pmiRGLO-KLF12-MUT) abolished the suppressive effect on luciferase activity (Fig. 3g). To determine whether there is an inverse correlation between miR-141 and KLF12 expression, the ovarian cancer cell lines OVCA433, $\mathrm{A} 2780 \mathrm{CP}$ and SKOV3 were transiently transfected with miR-141. The qPCR analysis confirmed the ectopic expression of miR-141 in the cell lines (1- to 30-fold), while a simultaneous reduction in KLF12 expression of 20 to $60 \%$ was observed (Fig. 3h). Moreover, western blotting also confirmed that the expression levels of KLF12 and miR141 were inversely correlated in miR-141 overexpressed OVCA433 and A2780CP cells, and miR-141depleted SKOV3 and OVCA433 cells using anti-miR ${ }^{\mathrm{m}}$ miRNA Inhibitor (Fig. 3i). These results suggest that miR141 regulates a network of genes or signaling inside the mammalian cells, while KLF12 is a direct downstream target of miR-141, and miR-141 could inhibit the expression of KLF12 mRNA and protein levels.

\section{KLF12 exerts a tumor-suppressive effect on ovarian cancer cells}

As there is an inverse relationship between KLF12 and miR-141, KLF12 may play an opposing role in ovarian cancer cell tumorigenicity and may have relatively low expression. Immunohistochemistry (IHC) was performed 
on the same human ovarian cancer tissue array used for the evaluation of miR-141 expression by ISH (OVC1021, Pantomics). Together with the ISH findings, the IHC results demonstrated that high expression of miR-141 was significantly associated with low expression of KLF12 $(N=97, P<0.001)$ (Fig. 4a) (Tables 1 and 2). Notably, clinicopathological analysis showed that both high expression of miR-141 ( $>5$-fold) and low expression of KLF12 ( $\leqq 5$-fold) were significantly correlated with latestage cancers $(P=0.008$ for high expression of miR-141 and $P=0.000$ for low expression of KLF12) and metastasis $(P=0.001$ for high expression of miR-141 and $P=0.01$ for low expression of KLF12) (Tables 1 and 2). Further study by analyzing Pan-Cancer miRNA-mRNA interaction maps from the Cancer Genome Atlas (TCGA) matched miRNA-Seq and RNA-Seq data using StarBase V2.0 [32, 33] (http://starbase.sysu.edu.cn/index.php), we found that the inverse correlation between miR-141 and KLF12 is not only observed in ovarian cancers but also other cancer types (11/14) (Additional file 1: Figure S3). This suggests that this inverse relationship between miR-141 and KLF12 is generally found in human cancers.

On the other hand, overexpression of KLF12 showed a 1- to 2-fold reduction in cell growth in the OVCA433 and SKOV3 cells cultured in low serum media (Fig. 4b), a 2-fold reduction in foci formation in OVCA433 cells (Fig. 3c), and a 5- to 7-fold reduction in colony formation in soft agar assays for OVCA433 and SKOV3 cells (Fig. 4d). In contrast, stable knockdown of KLF12 in two highly expressing KLF12 cell lines, A2780cp and OVCA433 (Fig. 4e), showed a 0.2 - to 2.5 -fold increase in cell growth (Fig. 4 f) and a $10 \%$ to $30 \%$ increase in foci formation (Fig. $4 \mathrm{~g}$ ). These findings demonstrate that KLF12 acts a downstream target of miR-141 and a tumor suppressor in ovarian cancers.

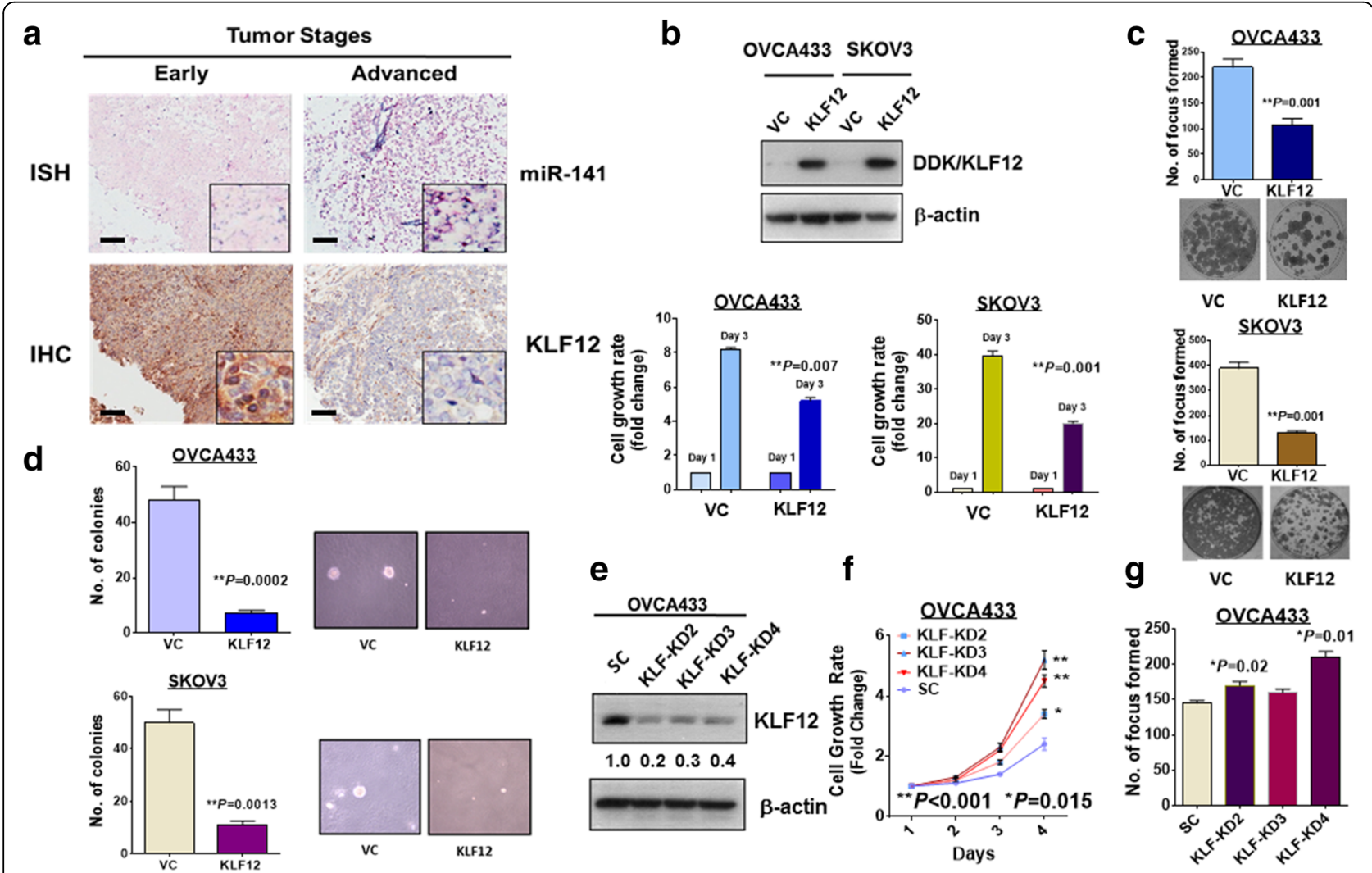

Fig. 4 KLF12 expression inversely correlates with miR-141 and exerts inhibitory effects on ovarian cancer tumorigenicity. a Representative pictures showing miR-141 expressions examined by ISH (dark blue staining) is inversely correlated with KLF12 expression detected by IHC (brown staining) in early and advanced ovarian cancers. (Magnification $\times 10$; scale bar, $100 \mu \mathrm{m}$ ). b Overexpression of KLF12 in two ovarian cancer cells; OVCA433 and SKOV3 (Upper), significantly suppresses their cell growth rates (Day 3 vs Day 1) examined by XTT cell proliferation assay when compared the cell growth rate of their empty vector controls, VC. c Focus formation assay revealed that overexpression of KLF12 significantly inhibit the number of foci in OVCA433 and SKOV3 cultured in low serum medium for 14 days. $\mathbf{d}$ The soft agar assay showed that overexpression of KLF12 remarkably reduce the number and size of colonies formed in soft agar in OVCA433 and SKOV3 cells as compared with their empty vector controls. The above assays represent the error bars with mean \pm SD of at least 3 independent experiments. e Western blot analysis revealed that three KLF12 stable clones were obtained from transfection of shRNAi of KLF12 in OVCA433. SC represents scrambled control. These results were obtained from at least three experiments. $\mathbf{f}$ XTT cell proliferation assay showed that there was a significant increase of cell growth rate in all three KLF12 knockdown clones as compared with the scramble control (SC). $\mathbf{g}$ The focus formation assay showed that there were two KLF12 knockdown clones (KD2 and KD4) had a significant increase in the number of foci 
Table 2 Clinicopathological and miR-141 correlation of the expression of an ovarian cancer tissue array (OVC1021). The 5-fold cut-off was determined by ROC analysis

\begin{tabular}{|c|c|c|c|c|}
\hline \multirow[t]{2}{*}{ Parameters } & \multirow[t]{2}{*}{$\mathrm{n}(=97)$} & \multicolumn{3}{|c|}{ KLF12 expression } \\
\hline & & $\leq 5$-fold & $>5$-fold & $p$ \\
\hline \multicolumn{5}{|l|}{ Grade } \\
\hline $\operatorname{Low}(1+2)$ & 50 & 25 (50\%) & $25(50 \%)$ & \\
\hline High (3) & 46 & $24(52 \%)$ & $22(48 \%)$ & 0.841 \\
\hline \multicolumn{5}{|l|}{ Stage } \\
\hline Early (1) & 48 & 14 (29\%) & 34 (71\%) & \\
\hline Late $(2+3)$ & 49 & 36 (73\%) & $13(27 \%)$ & $0.000^{*}$ \\
\hline \multicolumn{5}{|l|}{ Metastasis } \\
\hline Yes & 24 & 18 (75\%) & $6(25 \%)$ & \\
\hline No & 73 & 32 (44\%) & $41(56 \%)$ & $0.010^{*}$ \\
\hline \multicolumn{5}{|l|}{ miR-141 } \\
\hline$\leq 5$ folds & 50 & 14 (28\%) & $36(72 \%)$ & \\
\hline$>5$ folds & 48 & 37 (77\%) & $11(23 \%)$ & $0.000^{*}$ \\
\hline
\end{tabular}

miR-141 confers anoikis resistance by suppressing KLF12 in ovarian cancer cells

Given that miR-141 is involved in promoting resistance to the stress microenvironment in ovarian cancer cells, we next investigated whether miR-141 increases anoikis resistance in ovarian cancer cells. Anoikis is a type of apoptosis induced by inappropriate cell-matrix interactions [34], and the anoikis assay is a method that mimics the microenvironment of the cells when the extracellular matrix attachment is absent [35] (Fig. 5a). Upon pretreatment with the anoikis method, OVCA433 cells overexpressing miR-141 and with knockdown of KLF12 showed fewer apoptotic cells (red signals) (overexpressing miR-141, 3-fold; knockdown of KLF12, 3- to 8-fold) compared with their scrambled controls using TUNEL assays, indicating that there is a substantial reduction in apoptosis in OVCA433 cells (Fig. 5b). Flow cytometry analysis of KLF12 knockdown clones or miR-141-overexpressing clones further showed a reduction in the sub-G1 phase ( $28 \%$ reduction for KLF12 knockdown clones of OVCA433 and $\sim 56 \%$ for miR-141-overexpressing clones of SKOV3) (Fig. 5c), indicating less apoptosis in KLF12 knockdown OVCA433 cells or miR-141-overexpressing SKOV3 cells. Furthermore, western blotting revealed that expressions of apoptosis-related factors, cleaved-PARP and cleaved-caspase3, were reduced drastically (Fig. 5d). Collectively, the above results indicate that the overexpression of miR-141 not only increases cell viability in stress microenvironments but also confers anoikis resistance to the ovarian cancer cells by inhibiting KLF12.

To examine the functional role of miR-141 in metastatic peritoneal ovarian cancer growth and progression, miR-141 was stably expressed in SKOV3 clones $(N=4)$, and these cells and the vector controls $(N=4)$ were intraperitoneally (i.p.) injected into nude mice. After 4 weeks, tumor seeding and invasion were examined in a post-mortem examination. Although injection of both the vector control and the miR-141 clones resulted in tumor nodule formation, tumor seeding was observed in only three out of four mice in the vector control group while all four mice in the miR-141 group showed a greater extent of tumor burden. Moreover, compared with the vector control, cells with stably expressing miR141 had a greater number of macroscopic tumor nodules studding throughout the omentum and the peritoneal cavity. Each mouse injected with the miR-141 clones formed an average of more than 10 intraperitoneal tumor nodules, while there was an average of approximately 2 nodules in the vector control group (Fig. 5e). Taken together, these results further suggest that in addition to promoting anoikis resistance, miR-141 also promotes metastatic ovarian cancer cell growth and tumor seeding in the peritoneal cavity, which is important in the metastatic process.

\section{miR-141 serves as a novel modulator of the intrinsic apoptotic pathway}

To decipher the underlying molecular mechanism associated with miR-141/KLF12-mediated anoikis resistance, we examined their downstream targets. KLF12 is a transcription factor that belongs to the Sp1/KLF family [36]. Affymetrix GeneChip 3' expression arrays were used with mRNAs isolated from OVCA433 KLF12 stable knockdown clones (KLF-KD4) to identify the downstream targets regulated by KLF12 (Fig. 6a). Using a fold change $>2$ as a threshold, we identified a panel of differentially expressed genes, particularly those related to the apoptosis. Intriguingly, most highly altered genes revealed by array profiling analysis were associated with anti-apoptotic effects, e.g., CIAP2, Bcl2A1 and survivin (Fig. 6a), supporting the hypothesis that KLF12 is crucial in combating anoikis during cell detachment. Furthermore, using a Human Apoptosis Array Kit consisting of 35 apoptosis-related proteins (R\&D) with either miR-141overexpressing or KLF12 knockdown ovarian cancer cells, 8 out of 35 proteins (HO-2/HMOX2, ph-Rad 17, CIAP-2, survivin, HSP-70, TNFR1/TNFRSF1A, clusterin and XIAP) were upregulated (Fig. 6b). Upregulation of survivin was found in both GeneChip and the Human Apoptosis Arrays (Fig. 6b). Survivin, a structurally unique IAP protein, is a key regulator of the intrinsic apoptotic pathway. Previous findings reported that survivin forms a complex with XIAP and inhibits the activation of caspase3 and caspase-9, which in turn blocks the intrinsic apoptotic pathway [37] and prevents cell apoptosis due to anoikis stress. X-linked inhibitor of apoptosis protein (XIAP), also known as inhibitor of apoptosis protein 3 


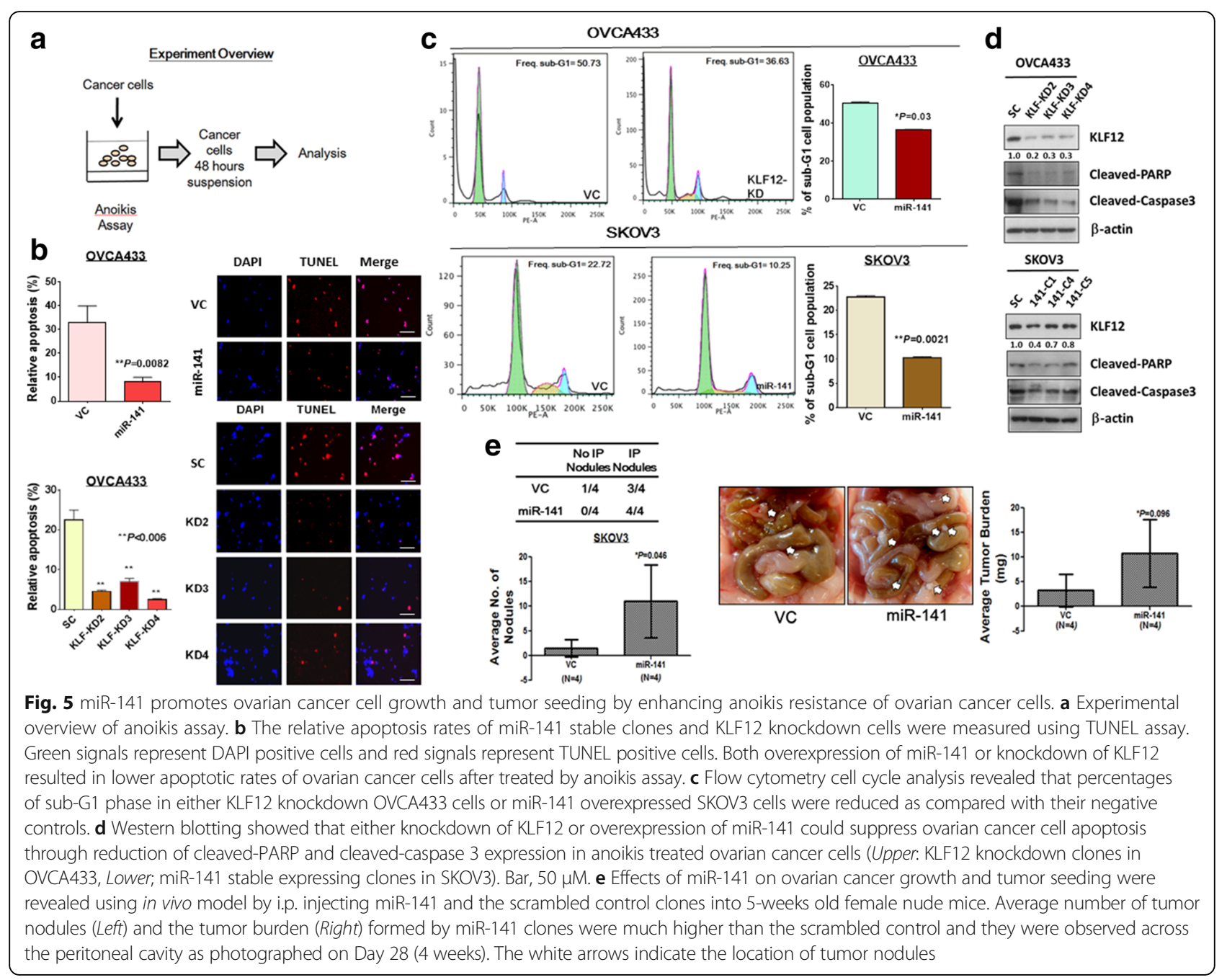

(IAP3), is also upregulated through association with the increased survivin [38]. Hence, we speculated that the miR-141-induced anoikis resistance is mediated through suppression of KLF12, which, in turn, upregulates survivin and XIAP expression to inhibit ovarian cancer cell apoptosis. Previous studies have suggested that the KLF family shares homology with the transcriptional factor Sp1, and therefore, they are grouped together in the Sp1/KLF family [39]. Thus, we hypothesized that KLF12 and Sp1, which share homology, may compete for binding sites on the survivin promoter, hampering the transcriptional activation of survivin (Fig. 6c). To test this hypothesis, we first used Sp1 motif from Jaspar database to search the Sp1binding elements (SEBs) on the survivin promoter (Fig. 6d). Taking the promoter region (+/ $-1 \mathrm{k}$ to TSS) of survivin: chr17:76209278-76211277, we found that ten putative SEBs (Additional file 4: Figure S4), showing higher potential of $\mathrm{Sp} 1$ binding according to recent findings [40]. By their affinity score and distribution on survivin promoter, 7 out of 10 binding sites were examined for the competition of SEBs occupancy between Sp1 and KLF12 by ChIP assay (Additional file 4: Figure S4). Results revealed that the occupancy of Sp1 on 3 out of $7 \mathrm{SBEs}$ of the survivin promoter were obviously inhibited by KLF12 in A2780cp ovarian cancer cells (Fig. 6d), supporting our notion of that KLF12 compete for the binding sites of $\mathrm{Sp} 1$ on the survivin promoter and in turns hampers the transcriptional activity of survivin.

To further prove that, we next examined whether the transcriptional activity of survivin is upregulated by $\mathrm{Sp} 1$ using luciferase reporter assays. pCMV6-Sp1 and a survivin promoter luciferase plasmid (luc-survivin) ( $\mathrm{CH} 3$ Biosystems) were co-transfected into HEK293 cells and A2780cp cells. The results showed that the luciferase activity of the survivin promoter was significantly increased by $\sim 3$ - to 5.5 -fold in HEK293 cells and by $\sim 48$ to $70 \%$ in $\mathrm{A} 2780 \mathrm{cp}$ cells by $\mathrm{Sp} 1$ in a dose-dependent manner (Fig. 6e). In contrast, when luc-survivin and pCMV6-Sp1 (600 ng) were co-transfected with varied amounts of pCMV6-KLF12 (200-800 ng) in HEK293 


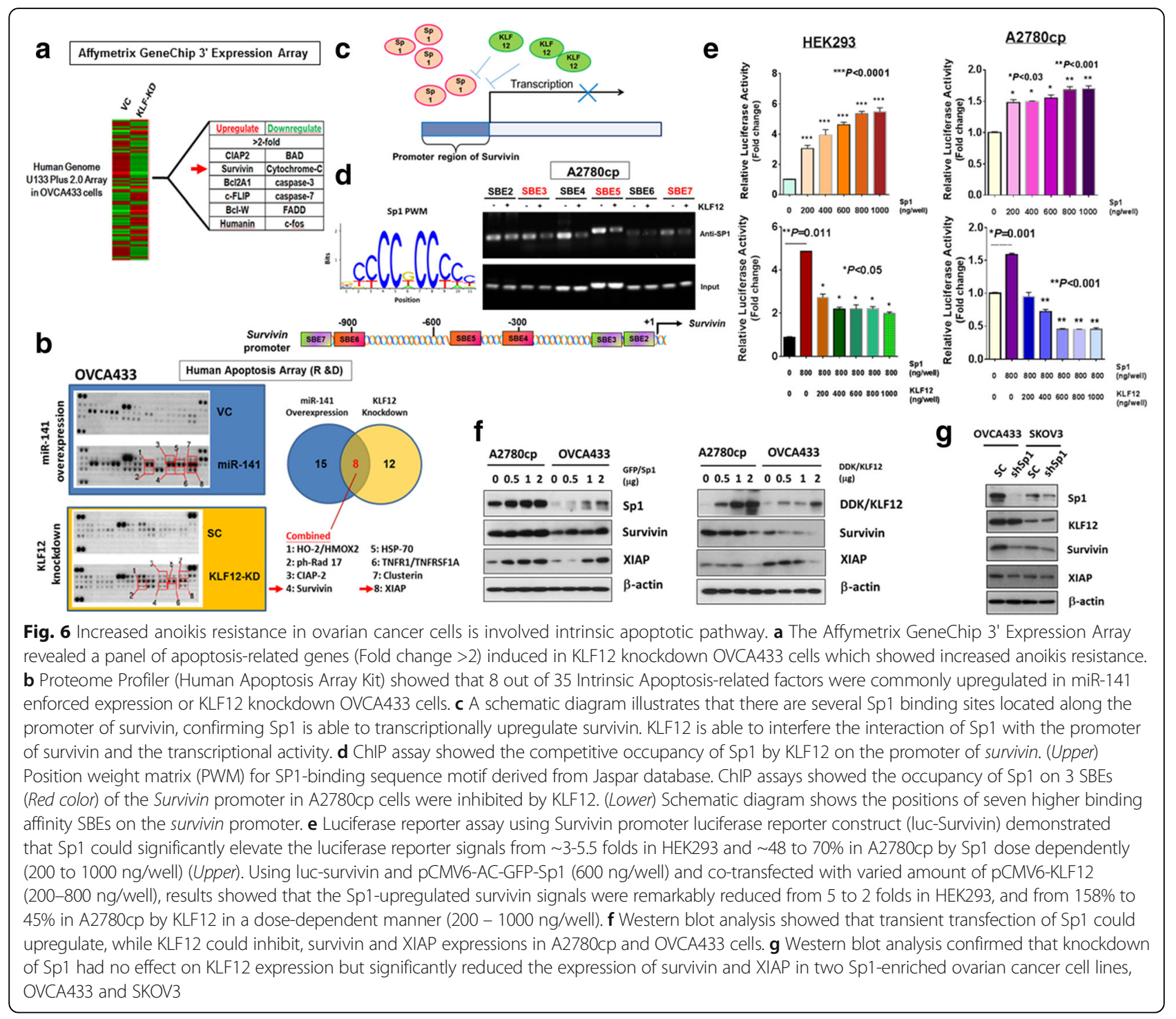

and A2780cp cells, the luciferase reporter results showed that the Sp1-upregulated survivin signals were strongly reduced by KLF12 from 5-fold to 2-fold in HEK293 cells and from $158 \%$ to $45 \%$ in A2780cp cells in a dosedependent manner (Fig. 6e). These results suggest that KLF12 has an opposing function to Sp1 and augments ovarian cancer cell survival by modulating the expression of survivin. To further verify these results, we transiently transfected A2780cp and OVCA433 cells with either Sp1 or KLF12. Western blot analyses indicated that $\mathrm{Sp} 1$ could significantly elevate the expression levels of survivin as well as XIAP, while KLF12 had the opposite effects compared to $\mathrm{Sp} 1$ and suppressed survivin and XIAP expressions in both ovarian cancer cell lines (Fig. 6f). In contrast, stable knockdown of Sp1 in OVCA433 and SKOV3 reduced the expression of survivin and XIAP, while the expression of KLF12 was not changed (Fig. 6g). These data suggest that the overexpression of miR-141 or the loss of KLF12 enhances anoikis resistance in ovarian cancer cells by modulating the Sp1/survivin/XIAP intrinsic apoptotic pathway.

\section{Survivin is required for enhancing anoikis resistance in ovarian cancer cells}

The above findings showed that the miR-141/KLF12/survivin signaling cascade is involved in the enhancement of anoikis resistance in ovarian cancer cells. We questioned whether survivin has a role in modulating anoikis resistance in ovarian cancer cells, although mounting evidence has indicated that survivin is frequently overexpressed in human cancers [41] and involved in chemoresistance [42] and metastatic progression [43]. We first knocked down survivin using a shRNA approach in two ovarian cancer cell lines, OVCA433 and SKOV3 (Fig. 7a). Foci formation assays revealed that depletion of survivin reduced the 

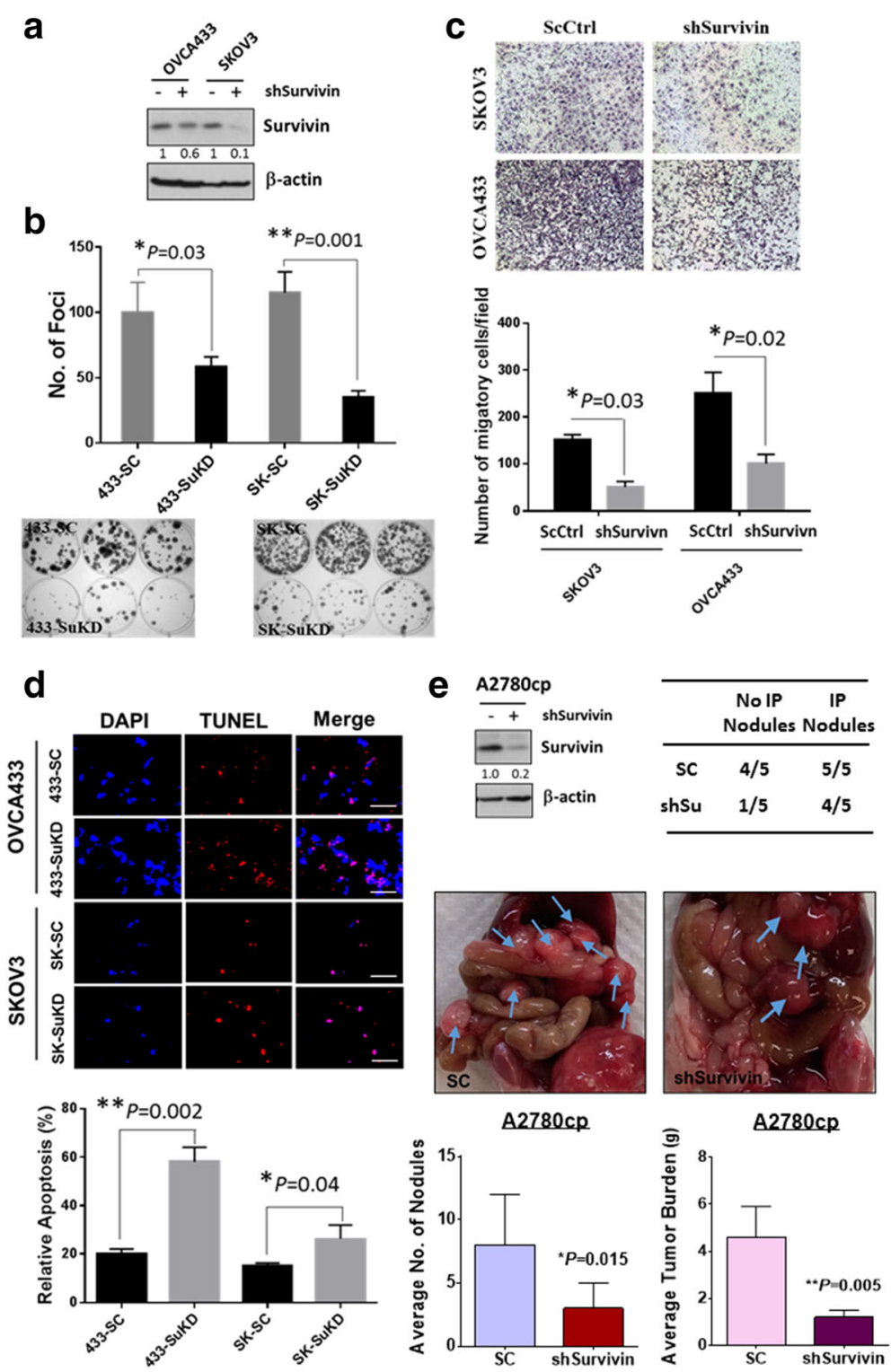

Fig. 7 Survivin is required for anoikis resistance, cell proliferation, cell migration and tumor colonization of ovarian cancer cells. a Western blot analysis revealed that stable knockdown of survivin clones were established in OVCA433 and SKOV3 cells. b Focus formation assay demonstrated that knockdown of survivin significantly reduce both number and size of focus. c Stable knockdown of survivin also significantly impaired the cell migration capacity of ovarian cancer cell lines; SKOV3 and OVCA433 cells. $\mathbf{d}$ Upon anoikis assay treatment, results showed that depletion of survivin by shRNAi remarkably increase the number of TUNEL positive signals (RED signals) as compared with their scrambled controls (SC), indicating there is increased apoptotic rates of survivin-knockdown ovarian cancer cells. Bar, $100 \mu \mathrm{M}$. e Effects of survivin knockdown on ovarian cancer colonization. Western blot analysis confirmed $80 \%$ survivin was knockdown by lentiviral shRNAi approach. Both ovarian cancer cells with stably knockdown of survivin and scrambled control (SC) of A2780cp were i.p. injected into 5-weeks old female nude mice. Average number of tumor nodules and the tumor burden formed by survivin knockdown clone were much lower than the scrambled control and the tumor nodules stunning throughout the peritoneal cavity as photographed on Day 21 (3 weeks). The blue arrows point out the position of tumor nodules

number of foci by $48 \%$ to $65 \%$ in these two cell lines (Fig. 7b). In addition, the cell migratory capacity of survivin knockdown SKOV3 and OVCA433 clones was reduced by 2.5 - to 3 -fold compared with their scrambled controls (Fig. 7c). Moreover, upon treatment with the anoikis assay, the depletion of survivin in these two cell lines caused a substantial increase in the cell apoptotic rate by 2 - to 3 -fold (Fig. $7 \mathrm{~d}$ ).

Finally, to examine the functional role of survivin in metastatic potential of ovarian cancer cell growth and progression in peritoneal cavity, survivin knockdown was performed in another ovarian cancer cell line; 
A2780cp has been shown to highly express survivin and displays highly tumorigenicity in nude mice (Fig. 7e) [44]. The stable A2780cp survivin knockdown cells (80\% knockdown) (Fig. 7e) $(N=5)$ and the vector control cells $(N=5)$ were intraperitoneally (i.p.) injected into nude mice, similar to the above in vivo tumorigenic assay, to examine the functional role of miR-141 in ovarian cancer cells. After 2 weeks, tumor growth was investigated by post-mortem examination. Compared with the scrambled control (SC), the survivin knockdown clone $(\mathrm{shSu})$ resulted in fewer tumor nodules; tumor growth was observed in only one out of five mice in the survivin knockdown (shSu) group compared with four of five mice in the scrambled control (SC) group (Fig. 7e). Moreover, compared with the scrambled control group, knockdown of survivin resulted in a substantial reduction in the number of macroscopic tumor nodules in the omentum and the whole peritoneal cavity. The tumor mass of the survivin knockdown clone was $\sim 4$-fold less than that observed in the scrambled control (Fig. 7e). Taken together, these results suggest that survivin is required for anoikis resistance and metastatic progression of ovarian cancer cells in peritoneal metastases.

\section{Discussion}

The acquisition of anoikis resistance in cancer cells in response to ECM detachment during cancer progression is responsible for the difficulties in treatment and unsatisfactory clinical management for different types of cancers. Thus far, the underlying molecular mechanisms of this process in cancer cells have received more attention recently, and many important findings have been reported $[45,46]$. However, studies examining how ovarian cancer cells escape anoikis during metastasis still remain rare. In this study, we showed that the upregulation of miR-141 and the subsequent suppression of KLF12 are essential for anoikis resistance in ovarian cancer cells both in vitro and in vivo. We also demonstrated that the expression of survivin mRNA and protein increased drastically when miR-141 was expressed ectopically or Sp1 was overexpressed. The robust expression of survivin and its downstream target, XIAP, serves as a bridge between miR-141 and inhibition of intrinsic apoptotic pathway. Knocking down KLF12 produced results similar to miR-141-induced anoikis resistance. But depletion of survivin by shRNAi approach remarkably reduced anoikis resistance of ovarian cancer cells and suppressed their tumor colonization in peritoneal cavity of mice, suggesting survivin is the major downstream effector of miR-141/KLF12. Importantly, the inverse correlation between miR-141 and KLF12 expression was clinically confirmed of that the miR-141/KLF12/Sp1/survivin is a new signaling axis required for anoikis resistance of ovarian cancers during metastatic progression.
The dysregulation of miRNAs has been shown to be associated with cancer progression $[47,48]$. The expression of the miR-200 family, including miR-141, is usually expressed differentially in various human cancers [49]. Previous studies have documented that the members of this family are downregulated and could suppresses epithelial-mesenchymal transition (EMT), while some members of this family are upregulated and could promote mesenchymal-epithelial transition (MET) in cancer development [50-52]. On the contrary, the miR-200 family in advanced and high-grade ovarian and breast cancers is frequently overexpressed and is closely related to distant metastasis [53, 54]. Indeed, the overexpressed miR-141 has been reported in association with increased chemoresistance and cell survival of ovarian cancer cells under oxidative stresses $[55,56]$. In line with these studies, we showed that miR-141 is significantly upregulated in ovarian cancer cell lines and advanced metastatic ovarian cancers. Importantly, the expression of miR-141 showed an increased stepwise along the tumor stages, indicating miR-141 plays a crucial role in promoting ovarian cancer progression. Previous findings have shown that the miR-200 family is well-known for their EMT-suppressive and MET-promoting functions in other human cancers $[57,58]$. However, little is known about their tumorpromoting abilities in metastatic ovarian cancers. In this study, a convergence of functional assays demonstrated that miR-141 not only elevates the viability of ovarian cancer cells in stressful microenvironments but also promotes anchorage independence, resulting in increased anoikis resistance in vitro and tumor growth and colonization in vivo. Anoikis resistance is the initial event and must be acquired by tumor cells when they undergo metastasis. In successful metastasis, anoikis resistance is important for cell survival in the circulation before the cells reach a distant location with a suitable ECM for reattachment to form a new niche. It is known that one miRNA could regulate a network of signaling through targeting a number of downstream targets [59]. In analysis across three well known miRNA target prediction programs; PicTar, miRanda and TargetScan 6.0, we found that there are 65 putative targets which can be further categorized into several main biological functions such as metabolic process, localization, cellular process, biological regulation, apoptotic process and cell survival. Intriguingly, some of the possible miR-141 targets are associated with cancer metastasis e.g. DLC1 [29], insulin receptor substrate 2 (IRS2) [30] and salt-inducible kinase 1 (SIK1) [31]. SIK1 is particularly of interest because SIK1 is able to interact with LKB1 which in turn, enhances p53-dependent anoikis and suppresses cancer metastasis [31]. However, our findings in this study showed that the overexpressed miR-141 could enhance anoikis resistance not only in p53-wildtype (OVCA433), but also in p53-mutated (A2780cp) and 
p53-deleted (SKOV3) ovarian cancer cell lines. That means there are other signalings governing anoikis resistance in ovarian cancers. Previous studies reported that some miR141 targets function in cell survival and biological process could enhance chemoresistance and cell survival such as FUS and PAPP-A $[27,28]$. These findings urged us to look into which possible targets in cell survival are involved in anoikis resistance of ovarian cancers. As expected, in combination of a series of functional and biochemical analyses, KLF12 is a direct target of miR-141 and importantly, the inverse relationship of KLF12 and miR-141 is significantly correlated with the advanced and metastatic ovarian cancers. KLF12 is a zinc finger protein that belongs to the relatively large SP/KLF family of transcription factors. The family consists of 25 members that regulate a wide range of cell functions, including different aspects of cancer progression. Most KLFs are upregulated in the early stages of cancer and are known to inhibit cancer cell invasiveness. However, the expression of EMT-suppressing KLFs is predominantly downregulated when the cells undergo systemic dissemination, which usually occurs in the advanced stages [60]. These findings support our clinicopathological analysis of the inverse correlation between miR-141 and KLF12 is related to cancer metastasis. Such inverse relationship between miR-141 and KLF12 is also confirmed in other human cancer types according to TCGA database. Furthermore, a recent study reported that KLF12 is a novel metastasis-suppressor gene and suppression of KLF12 resulted in anoikis resistance in lung cancer cells [61]. This suggests that miR-141 protects ovarian cancer cells from anoikis by suppressing KLF12 expression.

The study of anoikis is a new area in cancer research and thus, increasing evidence has suggested that the initiation and execution of anoikis is mediated by different pathways. The interplay between the intrinsic and extrinsic apoptotic pathways appears to be the most important. Although the recent study demonstrated KLF12 enhances anoikis resistance of lung metastatic cancer cells via regulating cell cycle progression [61], our findings in this study showed another mechanism of that the loss of KLF12 elevating the expression of survivin and its related intrinsic apoptotic pathway. Overexpression of survivin, which belongs to the inhibitor of apoptosis (IAP) proteins, may lead to cancer cell invasiveness, poor patient prognosis and low survival rates [62, 63]. The survivin anti-apoptotic network is mediated through the survivin-XIAP complex, which subsequently inhibits the activation of caspase- 9 and the cleavage of caspase 3 and PARP. Apart from being an apoptotic regulator, survivin also modulates cell cycle and proliferations [64]. Aberrant upregulation of survivin is frequently reported in many human cancers and involves in tumor growth, metastasis, chemoresistance and poor prognosis [64, 65]. Indeed, our in vitro and in vivo tumorigenic studies evidenced that survivin is required for anoikis resistance, tumor growth and cancer cell colonization in peritoneal metastases of ovarian cancer cells. This suggests that survivin is a crucial modulator of the intrinsic pathway in governing anoikis resistance of ovarian cancer cells.

Previous studies have suggested that the KLF family shares homology with the transcription factor Sp1, and therefore, they are grouped together as the Sp1/KLF family [39]. There are eight Sp1 binding sites with canonical or similar sequences [(G/T)(G/A)GGCG(G/T)(G/A)(G/A)(C/ $\mathrm{T})$ ] in the survivin promoter, and thus, Sp1 is a key transcription factor for controlling the expression of survivin [40]. Therefore, we hypothesized that KLF12 and Sp1, which share homology, may compete for binding sites in the survivin promoter, inhibiting the transcriptional activation of survivin. As expected, our ChIP assay demonstrated that some Sp1 binding sites on survivin promoter are interfered by KLF12. Consistently, luciferase reporter assays confirmed this model by showing a significant increase in the luciferase signals under the control of the survivin promoter when Sp1 was co-expressed, while a significant suppression was observed when KLF12 was co-transfected with Sp1. This finding was further verified by western blot analyses of ovarian cancer cells transiently transfected with SP1 and KLF12 or with a stable Sp1 knockdown. Taken together, our study revealed that the overexpression of miR-141 augments anoikis resistance in ovarian cancer cells by targeting and repressing the expression of KLF12, which, in turn, competes for binding sites in the survivin promoter with Sp1. The subsequent increase in survivin then protects ovarian cancer cells against anoikis by blocking the intrinsic apoptotic activity.

\section{Conclusions}

This study highlights a novel signaling cascade of miR$141 / \mathrm{KLF} 12 / \mathrm{Sp} 1 /$ survivin in promoting anoikis resistance of ovarian metastatic cancer cells and suggests a unique molecular mechanism by which the intrinsic apoptotic pathway is impeded in ovarian cancer tumors by microRNA-mediated epigenetic regulation during ovarian cancer metastatic progression (Additional file 5: Figure S5). Conceivably, targeting this signaling axis could form the basis for a novel therapeutic strategy to treat or even prevent ovarian cancer micrometastases.

\section{Additional files}

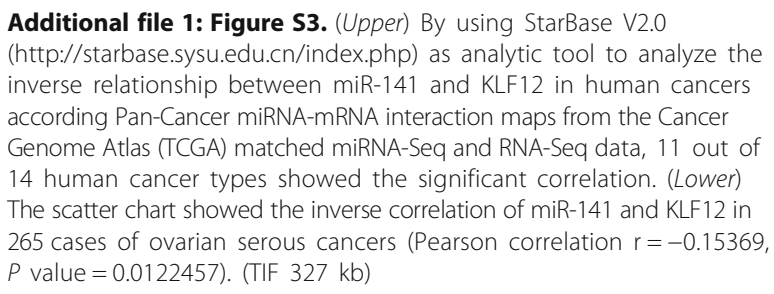


Additional file 2: Figure S1. Enforced expression of miR141 increases anchorage-independent growth capacity of ovarian cancer cells. Soft agar assay showed that stably expression of miR141 in A2780cp (141-C2 and 141-C8) and SKOV3 (141-C2 and 141-C3) could significantly increase the number and size of the colonies as compared with their vector control (VC). The pictures showed the representative colony morphology and size of miR141 stable clones and vector control (VC). (TIF $311 \mathrm{~kb}$ )

Additional file 3: Figure S2. Putative targets for miR-141. Schematic presentation of the miR-141 seed matches in the human KLF12, E2F3 and MAP2K4 3'UTR introduced into the pmiRGLO luciferase 3'UTR constructs. Luciferase reporter assay to assess the interaction of miR-141 with the three putative target candidates, E2F3, MAP2K4 and KLF12 with the use of HEK293 cells. Co-transfection of miR-141 and KLF12 luciferase plasmids resulted in the strongest reduction of the luciferase reporter signals. (TIF $3533 \mathrm{~kb}$ )

Additional file 4: Figure S4. (Upper) Taking the promoter region (+/-1 k to TSS) of survivin: chr17:76209278-76211277, ten putative SEBS higher potential of Sp1 binding ability were shown. (Lower) The genomic sequences of primers targeting SBES of Sp1 on the survivin promoter. (TIF $681 \mathrm{~kb}$ )

Additional file 5: Figure S5. A schematic diagram summaries the findings in this study. The overexpression of miR141 directly targets KLF12 expression. The reduced KLF12 expression allows Sp1 interacting with Survivin promoter that in turns, enhance the expression of survivin in both mRNA and protein levels. Through the stabilization of XIAP, the Survivin-XIAP complex which subsequently inhibiting the activation of caspase- 9 and the cleavage of caspase- 3 and PARP in the intrinsic apoptotic pathway and enhance anoikis resistance of advanced metastatic ovarian cancer cells in the ascetic tumor microenvironment. (TIF $320 \mathrm{~kb}$ )

\section{Acknowledgements}

We thank Prof. Benjamin Tsang (Department of Obstetrics and Gynecology, University of Ottawa) for providing ovarian cancer cell lines; OVCA433, A2780s, A2780cp, C13* and OV2008, and Prof. George Tsao (School of Biomedical Sciences) for HOSEs.

\section{Funding}

This study was supported by the Hong Kong Research Grants Council General Research Fund (17115115) and HKU Seed Funding Programme for Basic Research (201310159012).

\section{Availability of data and materials}

The cDNA microarray dataset and other data in the current study are available in https://figshare.com/s/6ee373748afd49babac6.

\section{Authors' contributions}

$C M$ and DC designed research; $C M, L H, M Y, L L, K C, R L, Y Q$ and DC performed the experiments; $\mathrm{DC}, \mathrm{TL}, \mathrm{KL}, \mathrm{KC}$ and $\mathrm{HN}$ contributed new reagents-analytic tools; CM, YQ, HN and DC analyzed and interpreted data; CM and DC wrote the manuscript; HN and DC study supervision. All authors were involved in editing the manuscript and had final approval of the submitted and published versions.

\section{Competing interests}

The authors declare that they have no competing interests.

\section{Consent for publication}

Not applicable.

\section{Ethics approval and consent to participate}

All clinical samples (49 ovarian cancer tissues and 12 normal ovarian tissues) were obtained from the Department of Obstetrics and Gynecology of The University of Hong Kong at Queen Mary Hospital. Written informed consent was provided by the participants and was approved by the Institutional Review Board of the University of Hong Kong/Hospital Authority Hong Kong West Cluster (HKU/HA HKW IRB) (Institutional Review Board number: UW05-143 T1806).

\section{Animal ethics}

All animal experiments were approved by the Committee on the Use of Live Animals in Teaching and Research of The University of Hong Kong (CULATR 3277-14).

\section{Author details}

'Department of Obstetrics and Gynaecology, L747 Laboratory Block, LKS Faculty of Medicine, The University of Hong Kong, 21 Sassoon Road, Hong Kong SAR, People's Republic of China. ${ }^{2}$ School of Biomedical Sciences, LKS Faculty of Medicine, The University of Hong Kong, 21 Sassoon Road, Hong Kong SAR, People's Republic of China.

Received: 5 September 2016 Accepted: 3 January 2017

Published online: 17 January 2017

\section{References}

1. Pereira A, Magrina JF, Magtibay PM, Perez-Medina T, Fernandez A, Peregrin I. The impact of peritoneal metastases in epithelial ovarian cancer with positive nodes. Int J Gynecol Cancer. 2011;21(8):1375-9.

2. Ursini-Siegel J, Siegel PM. The influence of the pre-metastatic niche on breast cancer metastasis. Cancer Lett. 2015;380(1):281-8.

3. Fidler IJ. The pathogenesis of cancer metastasis: the 'seed and soil' hypothesis revisited. Nat Rev Cancer. 2003;3(6):453-8.

4. Simpson $\mathrm{CD}$, Anyiwe K, Schimmer AD. Anoikis resistance and tumor metastasis. Cancer Lett. 2008;272(2):177-85.

5. Lu Z, Wang J, Wientjes MG, Au JL. Intraperitoneal therapy for peritoneal cancer. Future Oncol. 2010;6(10):1625-41.

6. Spring $B Q$, Abu-Yousif AO, Palanisami A, Rizvi I, Zheng X, Mai Z, Anbil S, Sears RB, Mensah LB, Goldschmidt R, et al. Selective treatment and monitoring of disseminated cancer micrometastases in vivo using dual-function, activatable immunoconjugates. Proc Natl Acad Sci U S A 2014;111(10):E933-942.

7. Lengyel E. Ovarian cancer development and metastasis. Am J Pathol. 2010;177(3):1053-64

8. Sugarbaker PH. Management of peritoneal metastases - Basic concepts. J BUON. 2015;20 Suppl 1:S2-11.

9. Lu YF, Zhang L, Waye MM, Fu WM, Zhang JF. MiR-218 mediates tumorigenesis and metastasis: perspectives and implications. Exp Cell Res. 2015;334(1):173-82.

10. Moss EG. MicroRNAs: hidden in the genome. Curr Biol. 2002;12(4):R138-140.

11. Pasquinelli AE, Reinhart BJ, Slack F, Martindale MQ, Kuroda MI, Maller B, Hayward DC, Ball EE, Degnan B, Muller P, et al. Conservation of the sequence and temporal expression of let-7 heterochronic regulatory RNA. Nature. 2000;408(6808):86-9.

12. Pasquinelli AE. MicroRNAs and their targets: recognition, regulation and an emerging reciprocal relationship. Nat Rev Genet. 2012;13(4):271-82.

13. Huntzinger $E$, Izaurralde $E$. Gene silencing by microRNAs: contributions of translational repression and mRNA decay. Nat Rev Genet. 2011;12(2):99-110.

14. Yang W, Lee DY, Ben-David Y. The roles of microRNAs in tumorigenesis and angiogenesis. Int J Physiol Pathophysiol Pharmacol. 2011;3(2):140-55.

15. Rao X, Di Leva G, Li M, Fang F, Devlin C, Hartman-Frey C, Burow ME, Ivan M, Croce CM, Nephew KP. MicroRNA-221/222 confers breast cancer fulvestrant resistance by regulating multiple signaling pathways. Oncogene. 2011;30(9):1082-97.

16. Garofalo M, Di Leva G, Romano G, Nuovo G, Suh SS, Ngankeu A, Taccioli C, Pichiorri $F$, Alder $H$, Secchiero $P$, et al. miR-221\&222 regulate TRAIL resistance and enhance tumorigenicity through PTEN and TIMP3 downregulation. Cancer Cell. 2009;16(6):498-509.

17. Sun $T$, Yang $M$, Chen $S$, Balk S, Pomerantz $M$, Hsieh CL, Brown M, Lee GS, Kantoff PW. The altered expression of MiR-221/-222 and MiR-23b/-27b is associated with the development of human castration resistant prostate cancer. Prostate. 2012;72(10):1093-103.

18. Quintavalle C, Garofalo M, Zanca C, Romano G, laboni M, Del Basso De Caro M, Martinez-Montero JC, Incoronato M, Nuovo G, Croce CM, et al. miR-221/222 overexpession in human glioblastoma increases invasiveness by targeting the protein phosphate PTPmu. Oncogene. 2012;31(7):858-68.

19. Lee JW, Park YA, Choi JJ, Lee YY, Kim CJ, Choi C, Kim TJ, Lee NW, Kim BG, Bae DS. The expression of the miRNA-200 family in endometrial endometrioid carcinoma. Gynecol Oncol. 2011;120(1):56-62.

20. Gregory PA, Bert AG, Paterson EL, Barry SC, Tsykin A, Farshid G, Vadas MA Khew-Goodall Y, Goodall GJ. The miR-200 family and miR-205 regulate 
epithelial to mesenchymal transition by targeting ZEB1 and SIP1. Nat Cell Biol. 2008;10(5):593-601.

21. Tsao SW, Mok SC, Fey EG, Fletcher JA, Wan TS, Chew EC, Muto MG, Knapp RC, Berkowitz RS. Characterization of human ovarian surface epithelial cells immortalized by human papilloma viral oncogenes (HPV-E6E7 ORFs). Exp Cell Res. 1995;218(2):499-507.

22. Liu MX, Siu MK, Liu SS, Yam JW, Ngan HY, Chan DW. Epigenetic silencing of microRNA-199b-5p is associated with acquired chemoresistance via activation of JAG1-Notch1 signaling in ovarian cancer. Oncotarget. 2014;5(4):944-58.

23. Nam EJ, Yoon H, Kim SW, Kim H, Kim YT, Kim JH, Kim JW, Kim S. MicroRNA expression profiles in serous ovarian carcinoma. Clin Cancer Res. 2008;14(9):2690-5.

24. da Huang W, Sherman BT, Lempicki RA. Systematic and integrative analysis of large gene lists using DAVID bioinformatics resources. Nat Protoc. 2009:4(1):44-57.

25. Kumar A, Wong AK, Tizard ML, Moore RJ, Lefevre C. miRNA_Targets: a database for miRNA target predictions in coding and non-coding regions of mRNAs. Genomics. 2012;100(6):352-6.

26. Loddo M, Andryszkiewicz J, Rodriguez-Acebes S, Stoeber K, Jones A, Dafou D, Apostolidou S, Wollenschlaeger A, Widschwendter M, Sainsbury R, et al. Pregnancy-associated plasma protein $A$ regulates mitosis and is epigenetically silenced in breast cancer. J Pathol. 2014;233(4):344-56.

27. Zhang Y, Chen B, Ming L, Qin H, Zheng L, Yue Z, Cheng Z, Wang Y, Zhang D, Liu C, et al. MicroRNA-141 inhibits vascular smooth muscle cell proliferation through targeting PAPP-A. Int J Clin Exp Pathol. 2015;8(11):14401-8.

28. Wang Z, Lei H, Sun Q. MicroRNA-141 and its associated gene FUS modulate proliferation, migration and cisplatin chemosensitivity in neuroblastoma cell lines. Oncol Rep. 2016;35(5):2943-51.

29. Wu PP, Zhu HY, Sun XF, Chen LX, Zhou Q, Chen J. MicroRNA-141 regulates the tumour suppressor DLC1 in colorectal cancer. Neoplasma. 2015;62(5):705-12.

30. Dong S, Meng X, Xue S, Yan Z, Ren P, Liu J. microRNA-141 inhibits thyroid cancer cell growth and metastasis by targeting insulin receptor substrate 2 . Am J Transl Res. 2016;8(3):1471-81.

31. Cheng H, Liu P, Wang ZC, Zou L, Santiago S, Garbitt V, Gjoerup OV, lglehart $J D$, Miron A, Richardson AL, et al. SIK1 couples LKB1 to p53-dependent anoikis and suppresses metastasis. Sci Signal. 2009;2(80):ra35.

32. Li JH, Liu S, Zhou H, Qu LH, Yang JH. starBase v2.0: decoding miRNA-ceRNA, miRNA-ncRNA and protein-RNA interaction networks from large-scale CLIP-Seq data. Nucleic Acids Res. 2014;42(Database issue):D92-97.

33. Yang JH, Li JH, Shao P, Zhou H, Chen YQ, Qu LH. starBase: a database for exploring microRNA-mRNA interaction maps from Argonaute CLIP-Seq and Degradome-Seq data. Nucleic Acids Res. 2011;39(Database issue):D202-209.

34. Frisch SM, Francis $\mathrm{H}$. Disruption of epithelial cell-matrix interactions induces apoptosis. J Cell Biol. 1994;124(4):619-26.

35. Guadamillas MC, Cerezo A, Del Pozo MA. Overcoming anoikis-pathways to anchorage-independent growth in cancer. J Cell Sci. 2011;124(Pt 19):3189-97.

36. Tetreault MP, Yang Y, Katz JP. Kruppel-like factors in cancer. Nat Rev Cancer. 2013;13(10):701-13.

37. Altieri DC. Survivin, cancer networks and pathway-directed drug discovery. Nat Rev Cancer. 2008;8(1):61-70.

38. Dohi T, Okada K, Xia F, Wilford CE, Samuel T, Welsh K, Marusawa H, Zou H, Armstrong R, Matsuzawa S, et al. An IAP-IAP complex inhibits apoptosis. J Biol Chem. 2004;279(33):34087-90.

39. Suske G, Bruford E, Philipsen S. Mammalian SP/KLF transcription factors: bring in the family. Genomics. 2005;85(5):551-6.

40. Mityaev MV, Kopantzev EP, Buzdin AA, Vinogradova TV, Sverdlov ED. Functional significance of a putative sp1 transcription factor binding site in the survivin gene promoter. Biochem Biokhim. 2008;73(11):1183-91.

41. Xia H, Chen $\mathrm{S}$, Huang $\mathrm{H}, \mathrm{Ma} \mathrm{H}$. Survivin over-expression is correlated with a poor prognosis in esophageal cancer patients. Clin Chim Acta. 2015;446:82-5.

42. Tsubaki M, Takeda T, Ogawa N, Sakamoto K, Shimaoka H, Fujita A, Itoh T, Imano M, Ishizaka T, Satou T, et al. Overexpression of survivin via activation of ERK1/2, Akt, and NF-kappaB plays a central role in vincristine resistance in multiple myeloma cells. Leuk Res. 2015;39(4):445-52.

43. Ye Q, Cai W, Zheng Y, Evers BM, She QB. ERK and AKT signaling cooperate to translationally regulate survivin expression for metastatic progression of colorectal cancer. Oncogene. 2014;33(14):1828-39.

44. Jiang Q, Dai L, Cheng L, Chen X, Li Y, Zhang S, Su X, Zhao X, Wei Y, Deng H. Efficient inhibition of intraperitoneal ovarian cancer growth in nude mice by liposomal delivery of short hairpin RNA against STAT3. J Obstet Gynaecol Res. 2013;39(3):701-9.
45. Paoli P, Giannoni E, Chiarugi P. Anoikis molecular pathways and its role in cancer progression. Biochim Biophys Acta. 2013;1833(12):3481-98.

46. Tan K, Goldstein D, Crowe P, Yang JL. Uncovering a key to the process of metastasis in human cancers: a review of critical regulators of anoikis. J Cancer Res Clin Oncol. 2013;139(11):1795-805.

47. Iorio MV, Visone R, Di Leva G, Donati V, Petrocca F, Casalini P, Taccioli C, Volinia S, Liu CG, Alder H, et al. MicroRNA signatures in human ovarian cancer. Cancer Res. 2007;67(18):8699-707.

48. Li M, Li J, Ding X, He M, Cheng SY. microRNA and cancer. AAPS J. 2010;12(3):309-17.

49. Feng $X$, Wang $Z$, Fillmore $R$, Xi Y. MiR-200, a new star miRNA in human cancer. Cancer Lett. 2014;344(2):166-73.

50. Ding XM. MicroRNAs: regulators of cancer metastasis and epithelial-mesenchymal transition (EMT). Chin J Cancer. 2014;33(3):140-7.

51. Katz L. Special issue dedicated to Sir David Alan Hopwood. J Ind Microbiol Biotechnol. 2014;41(2):173-477.

52. Chen J, Wang L, Matyunina LV, Hill CG, McDonald JF. Overexpression of miR-429 induces mesenchymal-to-epithelial transition (MET) in metastatic ovarian cancer cells. Gynecol Oncol. 2011;121(1):200-5.

53. Muralidhar GG, Barbolina MV. The miR-200 family: versatile players in epithelial ovarian cancer. Int J Mol Sci. 2015;16(8):16833-47.

54. Dykxhoorn DM, Wu Y, Xie H, Yu F, Lal A, Petrocca F, Martinvalet D, Song E, Lim B, Lieberman J. miR-200 enhances mouse breast cancer cell colonization to form distant metastases. PLoS One. 2009;4(9):e7181.

55. Mateescu B, Batista L, Cardon M, Gruosso T, de Feraudy Y, Mariani O, Nicolas A, Meyniel JP, Cottu P, Sastre-Garau X, et al. miR-141 and miR-200a act on ovarian tumorigenesis by controlling oxidative stress response. Nat Med. 2011;17(12):1627-35.

56. van Jaarsveld MT, Helleman J, Boersma AW, van Kuijk PF, van ljcken WF, Despierre E, Vergote I, Mathijssen RH, Berns EM, Verweij J, et al. miR-141 regulates KEAP1 and modulates cisplatin sensitivity in ovarian cancer cells. Oncogene. 2013;32(36):4284-93.

57. Koutsaki M, Spandidos DA, Zaravinos A. Epithelial-mesenchymal transitionassociated miRNAs in ovarian carcinoma, with highlight on the miR-200 family: prognostic value and prospective role in ovarian cancer therapeutics. Cancer Lett. 2014;351 (2):173-81.

58. Wang L, Mezencev R, Svajdler M, Benigno BB, McDonald JF. Ectopic over-expression of miR-429 induces mesenchymal-to-epithelial transition (MET) and increased drug sensitivity in metastasizing ovarian cancer cells. Gynecol Oncol. 2014;134(1):96-103.

59. Iwama $\mathrm{H}$. Coordinated networks of microRNAs and transcription factors with evolutionary perspectives. Adv Exp Med Biol. 2013;774:169-87.

60. Limame R, Op de Beeck K, Lardon F, De Wever O, Pauwels P. Kruppel-like factors in cancer progression: three fingers on the steering wheel. Oncotarget. 2014;5(1):29-48.

61. Godin-Heymann N, Brabetz S, Murillo MM, Saponaro M, Santos CR, Lobley A, East P, Chakravarty P, Matthews N, Kelly G, et al. Tumour-suppression function of KLF12 through regulation of anoikis. Oncogene. 2016;35(25): 3324-34.

62. Gu X, Lin HL. Analysis of survivin expression in subtypes of lymphoma Ai Zheng. 2004;23(6):655-61.

63. Sarela Al, Macadam RC, Farmery SM, Markham AF, Guillou PJ. Expression of the antiapoptosis gene, survivin, predicts death from recurrent colorectal carcinoma. Gut. 2000;46(5):645-50.

64. Chen X, Duan N, Zhang C, Zhang W. Survivin and tumorigenesis: molecular mechanisms and therapeutic strategies. J Cancer. 2016;7(3):314-23.

65. Chen L, Liang L, Yan X, Liu N, Gong L, Pan S, Lin F, Zhang Q, Zhao H, Zheng F. Survivin status affects prognosis and chemosensitivity in epithelial ovarian cancer. Int J Gynecol Cancer. 2013;23(2):256-63. 